\title{
Functional Subdomains within Scene-Selective Cortex: Parahippocampal Place Area, Retrosplenial Complex, and Occipital Place Area
}

\author{
Tolga Çukur, ${ }^{1,2,3}$ Alexander G. Huth, ${ }^{5}$ Shinji Nishimoto, ${ }^{4}$ and $\oplus^{\top}$ Jack L. Gallant ${ }^{5,6,7}$ \\ ${ }^{1}$ Department of Electrical and Electronics Engineering, ${ }^{2}$ Ulusal Manyetik Rezonans Araştırma Merkezi, Sabuncu Brain Research Center, and ${ }^{3}$ Neuroscience \\ Program, Bilkent University, Ankara 06800, Turkey, ${ }^{4}$ Graduate School of Frontier Biosciences, Osaka University, Osaka 565-0871, Japan, and ${ }^{5} \mathrm{Helen}$ Wills \\ Neuroscience Institute, ${ }^{6}$ Program in Bioengineering, and ${ }^{7}$ Department of Psychology, University of California, Berkeley, California 94720
}

Functional MRI studies suggest that at least three brain regions in human visual cortex - the parahippocampal place area (PPA), retrosplenial complex (RSC), and occipital place area (OPA; often called the transverse occipital sulcus) - represent large-scale information in natural scenes. Tuning of voxels within each region is often assumed to be functionally homogeneous. To test this assumption, we recorded blood oxygenation level-dependent responses during passive viewing of complex natural movies. We then used a voxelwise modeling framework to estimate voxelwise category tuning profiles within each scene-selective region. In all three regions, cluster analysis of the voxelwise tuning profiles reveals two functional subdomains that differ primarily in their responses to animals, man-made objects, social communication, and movement. Thus, the conventional functional definitions of the PPA, RSC, and OPA appear to be too coarse. One attractive hypothesis is that this consistent functional subdivision of scene-selective regions is a reflection of an underlying anatomical organization into two separate processing streams, one selectively biased toward static stimuli and one biased toward dynamic stimuli.

Key words: category representation; fMRI; OPA; PPA; RSC; scene; subdomain; tuning profile; voxelwise model

\section{Significance Statement}

Visual scene perception is a critical ability to survive in the real world. It is therefore reasonable to assume that the human brain contains neural circuitry selective for visual scenes. Here we show that responses in three scene-selective areas-identified in previous studies - carry information about many object and action categories encountered in daily life. We identify two subregions in each area: one that is selective for categories of man-made objects, and another that is selective for vehicles and locomotion-related action categories that appear in dynamic scenes. This consistent functional subdivision may reflect an anatomical organization into two processing streams, one biased toward static stimuli and one biased toward dynamic stimuli.

\section{Introduction}

Visual scene perception is critical for our survival in the real world. It is therefore reasonable to expect that the brain contains neural circuitry specialized for processing the wealth of information in natural scenes (Field, 1987; Vinje and Gallant, 2000; Bar,

\footnotetext{
Received Sept. 28, 2014; revised July 13, 2016; accepted July 28, 2016.

Author contributions: T.Ç., A.G.H., S.N., and J.L.G. designed research; T.Ç. performed research; T.Ç. and A.G.H. contributed unpublished reagents/analytic tools; T.Ç. analyzed data; T.Ç. and J.L.G. wrote the paper.

The work was supported in part by grants from the National Eye Institute (EY019684) and from the Center for Science of Information, an National Science Foundation Science and Technology Center, under Grant Agreement CCF-0939370. T.Ç.'s work was supported in part by a European Molecular Biology Organization Installation Grant (IG 3028), a Scientific and Technological Research Council of Turkey (TUBITAK) 2232 Fellowship (113C011), a Marie Curie Actions Career Integration Grant (PCIG13-GA-2013-618101), a TUBITAK 3501 Career Grant (114E546), and a Turkish Academy of Science Young Scientists Award Programme (TUBA-GEBIP) fellowship. We thank D. Stansbury, A. Vu, N. Bilenko, and J. Gao for their help in various aspects of this research.

The authors declare no competing financial interests.
}

2004; Geisler, 2008). At least three regions in the human brainthe parahippocampal place area (PPA), the retrosplenial complex (RSC), and the occipital place area (OPA) — produce larger blood oxygenation level-dependent (BOLD) responses to scenes than to isolated objects. These regions are therefore commonly considered to be involved in scene representation (Grill-Spector and Malach, 2004; Dilks et al., 2013). The anatomical locations of these regions are usually identified using functional localizers (Spiridon et al., 2006). Each region of interest (ROI) is localized by imposing a statistical threshold on the BOLD-response con-

Correspondence should be addressed to either of the following: Tolga Çukur, Department of Electrical and Electronics Engineering, Bilkent University, Ankara TR-06800, Turkey. E-mail: cukur@ee.bilkent.edu.tr; or Jack L. Gallant, 3210 Tolman Hall \#1650,University of California at Berkeley, Berkeley, CA 94720. E-mail: gallant@berkeley.edu DOI:10.1523/JNEUROSCI.4033-14.2016

Copyright $\odot 2016$ the authors $\quad 0270-6474 / 16 / 3610257-17 \$ 15.00 / 0$ 
trast between scenes versus single objects. This localizer approach implicitly assumes that all voxels within an ROI have similar visual selectivity and that each ROI is functionally homogeneous (Friston et al., 2006). However, recent reports suggest that subregions within the PPA may differ in their visual responsiveness (Arcaro et al., 2009), and that voxels within the PPA might have heterogeneous spatial-frequency selectivity (Rajimehr et al., 2011) and functional connectivity (Baldassano et al., 2013). These findings suggest that the PPA, and perhaps other sceneselective ROIs, might consist of several functional subdomains that represent different visual information in natural scenes.

It is challenging to assess visual representations in sceneselective areas because they are thought to respond to higherorder correlations among natural image features that cannot be easily decomposed (Lescroart et al., 2015). This difficulty has fueled ongoing debates about what specific types of information are represented in these areas (Nasr et al., 2011). Previous studies suggested that scene-selective areas might represent low-level information related to spatial factors (Epstein and Kanwisher, 1998; MacEvoy and Epstein, 2007; Park et al., 2007, 2011; Kravitz et al., 2011b) and texture (Cant and Goodale, 2011), high-level information related to scene categories (Walther et al., 2009; Stansbury et al., 2013), and/or contextual associations (Bar et al., 2008). Some evidence also suggests that the PPA, RSC, and OPA represent specific object categories (Reddy and Kanwisher, 2007; Macevoy and Epstein, 2009; Mullally and Maguire, 2011; Troiani et al., 2014). A recent voxelwise modeling study from our laboratory showed that some PPA voxels are selective for specific categories of inanimate objects in natural scenes (Huth et al., 2012). Furthermore, another voxelwise modeling study from our laboratory showed that the fusiform face area (FFA), another classical functional ROI that is also category-selective, consists of several functional subdomains with diverse tuning properties (Çukur et al., 2013b). Therefore, it is possible that scene-selective areas might also comprise distinct functional subdomains with different selectivity for object and action categories.

Here, we specifically assess the functional heterogeneity of representations in the PPA, RSC, and OPA. We first recorded BOLD signals evoked by a large set of natural movies. We then used voxelwise modeling (Huth et al., 2012; Çukur et al., 2013a) to determine how thousands of distinct object and action categories were represented in single voxels located within each of these three ROIs. Finally, we performed cluster analysis on the measured category-tuning profiles to determine whether there are functional subdomains with diverse tuning properties within each ROI.

\section{Materials and Methods}

Subjects. Six healthy human subjects (S1-S6; mean age, $26.7 \pm 3.1$ years; five males; one female) with normal or corrected-to-normal vision participated in the study. The study consisted of five separate scan sessions: three sessions for the main experiment and two sessions for functional localizers. The protocols for these experiments were approved by the Committee for the Protection of Human Subjects at the University of California, Berkeley (UCB). Written informed consent was obtained from all subjects before scanning.

Main experiment. The main experiment was conducted in three separate sessions. During each session, whole-brain BOLD responses were recorded while subjects passively viewed a distinct selection of color natural movies. Potential stimulus biases were minimized by selecting the movies from a diverse set of sources as described by Nishimoto et al. (2011). High-definition movie frames were cropped to a square aspect ratio and down-sampled to $512 \times 512$ pixels $\left(24 \times 24^{\circ}\right.$; the entire movie stimulus used as stimuli in this study is available at http://crcns.org/ data-sets/vc/vim-2/about-vim-2). Subjects maintained steady fixation on a color $\operatorname{dot}\left(0.16 \times 0.16^{\circ}\right)$ superimposed onto the movies and located at the center of the visual field. The color of the dot changed at $3 \mathrm{~Hz}$ to ensure continuous visibility. Stimulus presentation was performed with an MRI-compatible projector (Avotec), a custom-built mirror system, and custom-designed presentation scripts.

Two separate datasets were acquired for training and testing voxelwise models. The training and test runs contained different natural movies, and the presentation order of these runs was interleaved during each scan session. A total of 12 training runs and 9 testing runs were acquired across the three sessions. A single training run lasted $10 \mathrm{~min}$ and was compiled by concatenating distinct $10-20 \mathrm{~s}$ movie clips presented without repetition. A single testing run was compiled by concatenating 10 separate 1 min blocks in random order. Each 1 min block was presented nine times across three sessions and evoked BOLD responses were averaged across these repeats. To minimize the effects of hemodynamic transients during movie onset, data collected during the initial $10 \mathrm{~s}$ of each run were discarded. These procedures resulted in a total of 3600 and 270 data samples for training and testing, respectively.

Note that these same data were analyzed in several recent studies from our laboratory (Huth et al., 2012; Çukuret al., 2013a,b). Huth et al. (2012) reported that category selectivity is organized in broad gradients distributed across the high-level visual cortex, and that some PPA voxels are selective for inanimate objects. However, that study did not systematically examine the variability and spatial organization of tuning for nonscene categories within individual scene-selective ROIs. The work of Çukur et al. (2013a) involved a study of selective attention with aims unrelated to those of the present study. In a separate study, Çukur et al. (2013b) discovered several functional subdomains within the FFA that showed differences in category tuning.

Functional localizers. Functional localizer data were acquired independently from the main experiment. Localizers for category-selective brain areas consisted of six 4.5 min runs of 16 blocks. Each block lasted $16 \mathrm{~s}$ and contained 20 static images randomly selected from one of the following categories: objects, scenes, faces, body parts, animals, and spatially scrambled objects (Spiridon et al., 2006). The presentation order of the category blocks was randomly shuffled across runs. Within a block, each image was flashed for $300 \mathrm{~ms}$, followed by a $500 \mathrm{~ms}$ blank period. To maintain vigilance, subjects were required to press a button when they detected two identical consecutive images. The localizer for retinotopically organized early visual areas consisted of four 9 min runs containing clockwise rotating polar wedges, counter-clockwise rotating polar wedges, expanding rings, and contracting rings, respectively (Hansen et al., 2007). The localizer for the intraparietal sulcus consisted of one 10 min run of 30 blocks. Each block lasted $20 \mathrm{~s}$ and contained either a self-generated saccade task (among a pattern of targets) or a resting task (Connolly et al., 2000). The localizer for the human motion processing complex $(\mathrm{MT}+)$ consisted of four $90 \mathrm{~s}$ runs of 6 blocks. Each block lasted $15 \mathrm{~s}$ and contained either continuous or temporally scrambled natural movies (Tootell et al., 1995).

MRI parameters. Data collection was performed at UCB using a $3 \mathrm{~T}$ Siemens Tim Trio MRI scanner (Siemens Medical Solutions) and a 32channel receiver array. $\mathrm{T}_{2}^{*}$-weighted functional data were collected using a gradient-echo echo-planar imaging sequence with the following parameters: $\mathrm{TR}=2 \mathrm{~s} ; \mathrm{TE}=31 \mathrm{~ms}$; a water-excitation pulse with flip angle of $70^{\circ}$; voxel size, $2.24 \times 2.24 \times 3.5 \mathrm{~mm}^{3}$; field-of-view, $224 \times 224 \mathrm{~mm}^{2}$; and 32 axial slices for whole-brain coverage. Anatomical data were collected using a $\mathrm{T}_{1}$-weighted magnetization-prepared rapid-acquisition gradient-echo sequence with the following parameters: $T R=2.30 \mathrm{~s}$, $\mathrm{TE}=3.45 \mathrm{~ms}$, flip angle $=10^{\circ}$, voxel size $=1 \times 1 \times 1 \mathrm{~mm}^{2}$, field-ofview $=256 \times 256 \times 192 \mathrm{~mm}^{3}$.

Data preprocessing. Functional brain volumes acquired in individual scan sessions were first motion-corrected and then aligned to the first session of the main experiment using Oxford Centre for Functional MRI of the Brain's Linear Image Registration Tool (Jenkinson et al., 2002). For each run, the low-frequency drifts in BOLD responses of individual voxels were removed using a median filter over a $120 \mathrm{~s}$ temporal window. The resulting time courses were normalized to have zero mean and unity SD. After temporal detrending, no temporal or spatial smoothing was 

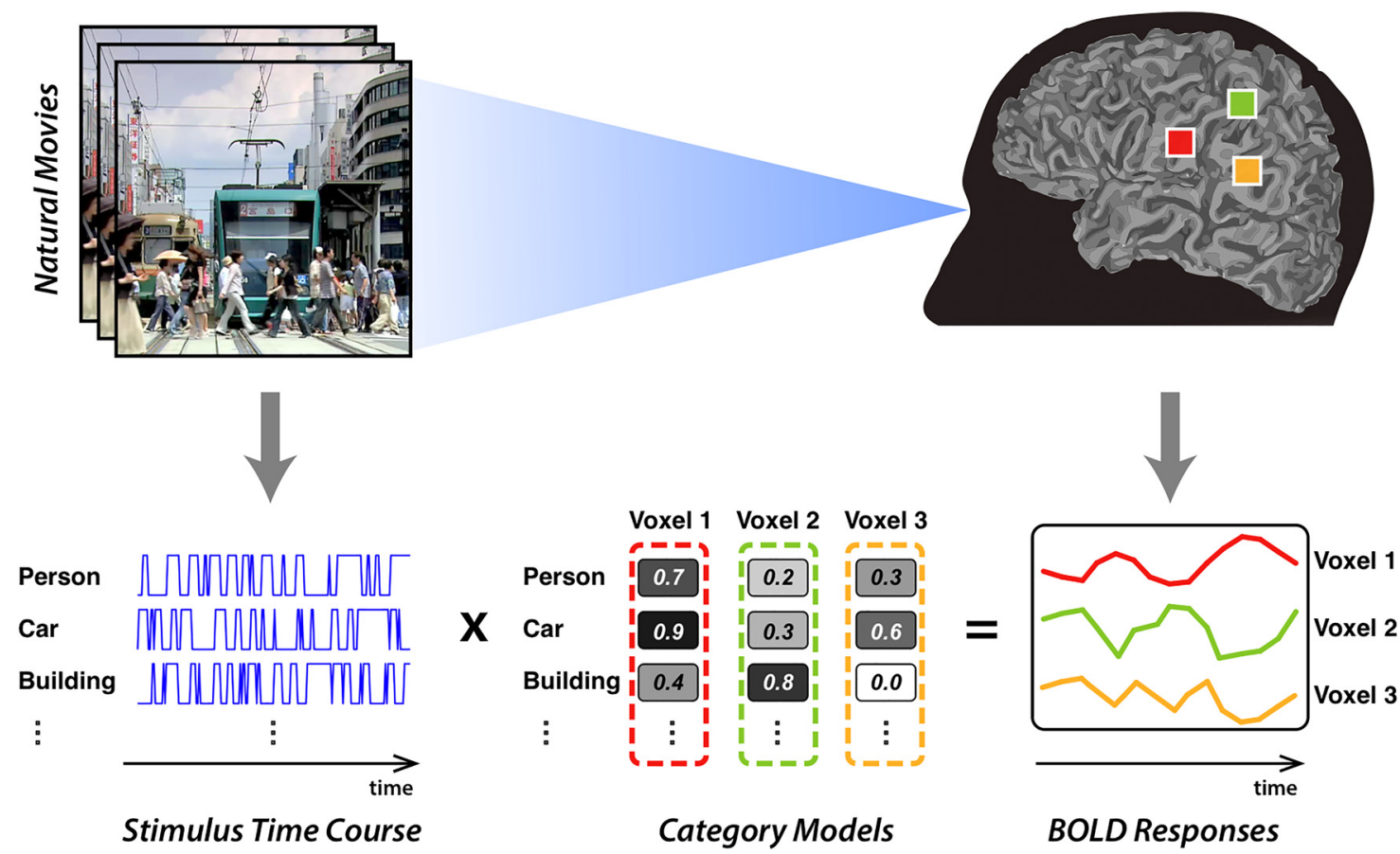

Figure 1. Voxelwise category models. A voxelwise modeling framework was used to measure category selectivity in single voxels from individual subjects. The WordNet lexicon was used to label salient object and action categories in each $1 \mathrm{~s} \mathrm{segment} \mathrm{of} \mathrm{the} \mathrm{movies} \mathrm{(Miller,} \mathrm{1995).} \mathrm{This} \mathrm{labeling} \mathrm{procedure} \mathrm{produced} \mathrm{for} \mathrm{each} \mathrm{category} \mathrm{a} \mathrm{separate} \mathrm{binary} \mathrm{variable} \mathrm{that} \mathrm{indicates} \mathrm{its} \mathrm{presence/absence}$ throughout the stimulus. The binary variables for 1705 distinct categories were taken as the stimulus features. Regularized linear regression was used to find a weighted sum of stimulus features that best describe the measured BOLD responses. The resulting model weights characterize the selectivity of single voxels to 1705 distinct object and action categories.

applied to the functional data from the main experiment. Functional localizer data were also motion-corrected and aligned to the first session of the main experiment. Following standard procedures, the localizer data were smoothed with a Gaussian kernel of full-width at halfmaximum equal to $4 \mathrm{~mm}$ (Spiridon et al., 2006).

Definition of functional ROIs. Category-selective ROIs were functionally defined in individual subjects using standard procedures (Spiridon et al., 2006). All scene-selective ROIs were defined from voxels with positive scene-versus-object contrast ( $t$ test, $p<10^{-4}$, uncorrected). The PPA was defined as the contiguous cluster of voxels in the parahippocampal gyrus, the RSC was defined as the contiguous cluster of voxels in the retrosplenial sulcus, and the OPA was defined as the contiguous cluster of voxels in the temporal-occipital sulcus with positive contrast. Additional category-selective regions, including the FFA, extrastriate body area, and lateral occipital complex, were defined using face-versus-object, body part-versus-object, and object-versus-scrambled-object contrasts.

Retinotopically organized early visual areas (V1-V4, V3a/b, and V7) were defined using standard retinotopic mapping techniques (Engel et al., 1997; Hansen et al., 2007). Last, the intraparietal sulcus area was defined as the contiguous cluster of voxels in the intraparietal sulcus that yielded positive saccade-versus-rest contrast ( $t$ test, $p<10^{-4}$, uncorrected). Area MT + was defined as the contiguous cluster of voxels in lateral-occipital lobe that yielded positive continuous-versus-scrambledmovie contrast ( $t$ test, $p<10^{-4}$, uncorrected).

Voxelwise encoding models. Separate voxelwise encoding models were fit to data from the main experiment to measure tuning for object and action categories, for spatial structure of visual scenes, and for elementary visual features. Each encoding model comprised a basis set of visual features (e.g., hundreds of distinct object categories) hypothesized to be represented in cortical voxels. The first step in building a voxelwise model is to quantify the time course of individual features across the movie stimulus. This was achieved by projecting the stimulus separately onto each feature in the basis set. Taking stimulus projections onto the model features as explanatory variables, encoding models were then fit to best predict measured BOLD responses. These quantitative models represent weighted linear combinations of features that best describe the relationship between natural movies and evoked BOLD responses. Therefore, the model weights for each voxel represent its selectivity for individual features in the basis set. The following sections describe the model bases and the regression procedures used to fit the models.

Category model. A primary goal of the study reported here is to assess category tuning within single voxels comprising scene-selective ROIs. To accomplish this, we used a voxelwise category model that was previously shown to accurately predict BOLD responses in high-level visual cortex (Huth et al., 2012; Çukur et al., 2013a). The basis set for this category model contained 1705 distinct object and action categories present in the natural movie stimulus. Using terms from the WordNet lexicon (Miller, 1995), the salient categories were manually labeled as present or absent. WordNet contains a semantic taxonomy that was used to infer the presence of more general categories. For example, a scene labeled with "baby" must contain a "human," a "living organism," and so on. Scene labels were assigned for every second of the movies, and aggregated across the stimulus to find the time courses for all model features (i.e., categories) as shown in Figure 1. Each time course was then temporally downsampled to $0.5 \mathrm{~Hz}$ to match the fMRI sampling rate. To reduce spurious correlations between global motion-energy and visual categories, a nuisance regressor was included that characterized the time course of total motion energy in the movie stimulus. Total motion energy was calculated as the summed output of all spatiotemporal Gabor filters used in the motionenergy model.

Gist model. One common view of scene-selective ROIs is that they represent information about the spatial structure of visual scenes. To measure selectivity for spatial texture and layout in single voxels, we fit a separate gist model. The gist model has been shown to provide a good account of spatial factors important for scene recognition, such as naturalness, expansion, and openness (Oliva and Torralba, 2001). Gist alone can be used to accurately distinguish scenes that belong to several different high-level categories. The features of the gist model were extracted by first spatially downsampling the movie stimulus to $256 \times 256$ pixels. A total of 512 model features were then calculated across eight orientations per scale and four spatial scales, where each scale was divided into $4 \times 4$ blocks. Finally, the time courses for all features were temporally downsampled to $0.5 \mathrm{~Hz}$ to match the fMRI sampling rate. 
Motion-energy model. Many voxels throughout the visual system are selective for elementary visual features, such as spatial location or spatiotemporal frequency. To measure selectivity for elementary features in single voxels, a motion-energy model was fit that was previously shown to accurately predict BOLD responses to natural movies in retinotopically organized early visual areas (Nishimoto et al., 2011). This motionenergy model contained 2139 spatiotemporal Gabor filters. Each filter was a three-dimensional spatiotemporal sinusoid multiplied by a spatiotemporal Gaussian envelope. Filters were computed at six spatial frequencies $(0,1.5,3,6,12$, and 24 cycles/image), three temporal frequencies $(0,2$, and $4 \mathrm{~Hz})$, and eight directions $(0,45,90,135,180,225$, 270 , and $\left.315^{\circ}\right)$. Filters were positioned on a square grid that spanned $24 \times 24^{\circ}$. Filters at each spatial frequency were placed on the grid such that adjacent filters were separated by a distance of 4 SDs of the spatial Gaussian envelope.

Model fitting. All voxelwise models were fit using regularized linear regression with an $\mathrm{l}_{2}$-penalty on model weights to prevent overfitting. The temporal sampling rates of the stimulus and BOLD responses were matched by down-sampling the stimulus time course twofold. Hemodynamic response functions were modeled separately for each model feature using separate linear finite-impulse-response (FIR) filters. FIR filter delays were restricted to $4-8 \mathrm{~s}$ (equivalently $2-4$ samples), and FIR coefficients were fit simultaneously with model weights to obtain highquality fits.

A 10-fold cross-validation procedure was used to optimize model weights to predict BOLD responses in the training data (Fig. 1). In each fold, $10 \%$ of the training data were randomly held out, and the models were fit to the remaining data. Model performance was assessed on the held-out data by calculating prediction scores, i.e., the correlation coefficient (Pearson's $r$ ) between the actual and predicted BOLD responses. The optimal regularization parameter for each voxel was determined by maximizing its prediction score. Finally, the optimal parameters were used to refit the models to all training data in a single step.

Model performance was assessed on independent test data using a jackknifing procedure. BOLD response predictions on the test data were randomly resampled 10,000 times without replacement (at a rate of $80 \%)$. Model performance was measured as the average prediction score across jackknife iterations. Model fitting was performed using custom software written in Matlab (MathWorks). When necessary, significance levels were corrected for multiple comparisons using false-discovery-rate control (Benjamini and Yekutieli, 2001).

Variance partitioning analysis. Objects and actions in natural movies can be correlated with lower-level visual features. It is therefore possible that the category models estimated here might be biased by selectivity for low-level features in scene-selective ROIs. To check for this potential confound, we performed a variance partitioning analysis. This analysis corrects the response variance explained by the category model to account for variance that can be attributed to low-level features captured by the gist or motion-energy models. To do this, we separately measured the variance explained when all three models (category, gist, and motion energy) are fit simultaneously, the variance explained when two models are fit simultaneously, and the variance explained by regressors of individual models. The proportion of variance for each model was calculated with respect to the variance explained by the simultaneous fit of all three models. Leveraging simple set-theoretic relations among the measurements, we extracted the proportion of unique variance explained by each model, and the proportion of shared variance explained commonly by multiple models.

Cluster analysis. The core issue that we address in this report concerns whether category selectivity is heterogeneous across voxels located within scene-selective ROIs. To investigate this issue, we performed separate cluster analyses on voxelwise tuning profiles measured within the PPA, $\mathrm{RSC}$, and OPA. The analyses were first run at the group level by pooling tuning profiles in each ROI across subjects. This group analysis yields common cluster labeling and facilitates comparisons among subjects. To ensure that the group clusters were consistent at a single subject level, cluster analyses were also repeated in individual subjects. The cluster solutions were compared by calculating the correlation coefficient between the obtained cluster centers.
We examined the group structure among ROI voxels using a sensitive spectral-clustering algorithm ( $\mathrm{Ng}$ et al., 2001). The dissimilarity between pairs of tuning profiles was characterized by a normalized Euclideandistance measure. To determine the number of clusters in the data, we used an unsupervised stability-based validation method (Ben-Hur et al., 2002; Handl et al., 2005). This validation method repeats the clustering analyses for a given number of clusters on random subsamples of the data. The stability for a given number of clusters is measured as the similarity between the cluster solutions on different subsamples. By repeating this procedure many times, the empirical probability distribution of clustering stability is obtained. If the number of clusters is appropriate for the data, then the cluster solutions should be stable. In contrast, if a suboptimal number is chosen, then the cluster solutions should be unstable.

Here we estimated the probability distribution of clustering stability by a random subsampling procedure repeated 5000 times. To enhance sensitivity, this procedure was performed after pooling voxels within each area across subjects. During each repeat, $80 \%$ of voxels were randomly selected without replacement twice, and the cluster solutions of this pair of subsamples were compared. The similarity of the solutions was quantified using the Jaccard Index (Jaccard, 1908). The cumulative distribution of clustering stability was estimated using normalized histograms (for a bin width of 0.005 ) across 5000 repeats. In this analysis, distributions of stable cluster solutions will be concentrated around unity similarity values, whereas distributions of unstable solutions will be more variable. For this reason, we determined the optimal number of clusters by comparing the value of the cumulative distribution functions at a high stability threshold for different numbers of clusters (Ben-Hur et al., 2002; Çukur et al., 2013b). A stability threshold of 0.9 was used here based on previously suggested values (Ben-Hur et al., 2002), but similar results were obtained for threshold values in the $0.80-0.95$ range.

Functional importance of heterogeneous selectivity. Here we assessed heterogeneous selectivity in scene-selective ROIs in two steps: we first fit encoding models to measure category selectivity in individual voxels; we then clustered the model weights to identify subdomains within each ROI. We performed two complementary analyses to evaluate both the functional importance of intervoxel differences in selectivity and intercluster differences in selectivity. First we asked whether individual voxels in each ROI show significant heterogeneity that would justify a cluster analysis. We reasoned that if model weights are significantly different across voxels, then a model fit to an individual voxel (self-prediction) should explain more of that voxel's responses than is explained by models fit to other voxels within the ROI (cross-prediction). We thus compared self-prediction and cross-prediction in terms of the proportion of variance explained in held-out test data.

Next we asked whether the voxel clusters within each ROI show functionally important differences in selectivity. If selectivity is significantly different across clusters, then a target voxel's responses should be better explained by models fit to other voxels in the same cluster (within-cluster prediction) than it is by models fit to voxels in a different cluster (crosscluster prediction). Therefore we compared within-cluster and crosscluster prediction in terms of proportion of explained variance. This analysis was repeated by obtaining separate predicted responses using category, gist, and motion-energy models. In both analyses of heterogeneity, the proportion of variance for each model was calculated with respect to the variance explained by the simultaneous fit of all three models. Significant differences were assessed with bootstrap tests.

Visualization of cluster centers. To interpret differences between the cluster centers, we visualized the mean tuning profile of each cluster within its optimal model space. For the category model, a graphical tree was constructed to visualize category responses to distinct objects and actions. The vertices of the graph corresponded to 1705 distinct categories. The connecting edges of the graph represented the hierarchical relationships between these categories as given by WordNet. The size and color of vertices represented the magnitude and sign of the category responses, respectively. For the motion-energy model, line plots were used to visualize the responses to distinct spatiotemporal frequencies.

Visualization on cortical surfaces. To understand the spatial organization of subregions within classical scene-selective ROIs, we projected 
a

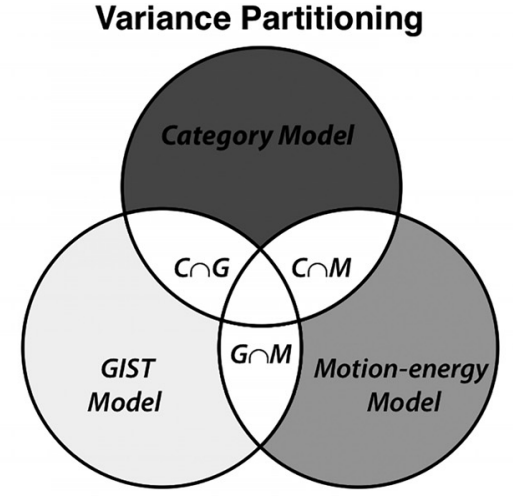

b

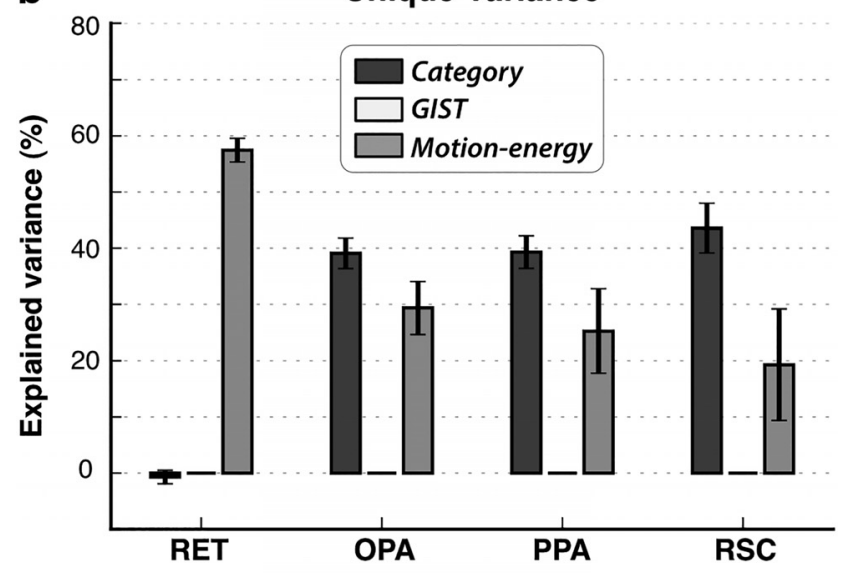

C

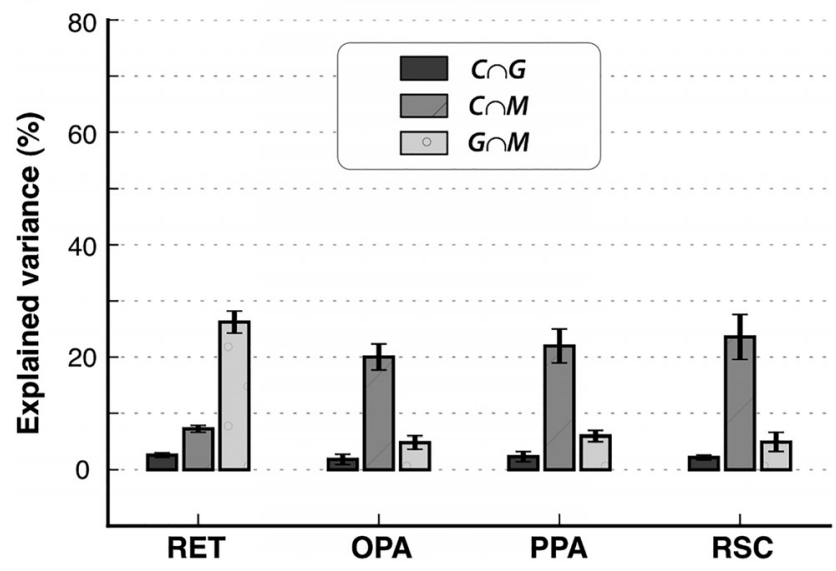

Figure 2. Selectivity for object and action categories. $\boldsymbol{a}$, Three separate models were estimated for each voxel: a category model that describes selectivity for object and action categories; a gist model that describes selectivity for spatial structures of scenes; and a motion-energy model that describes selectivity for low-level visual features. Models were validated by predicting BOLD responses in a separate dataset reserved for this purpose. A variance partitioning analysis was used to estimate the proportion of response variance predicted uniquely by each model and jointly by multiple models (see diagram). $\boldsymbol{b}$, The proportion of variance explained uniquely by category, gist, and motion-energy models in each ROI (mean \pm SEM across subjects). In early retinotopic visual areas (RET), the category model does not explain variance beyond what can be attributed to selectivity for spatial structure or low-level visual features. In contrast, the category model explains a significant portion of variance in scene-selective ROls ( $p<10^{-4}$, bootstrap test). This result suggests that selectivity for nonscene categories in the PPA, RSC, and OPA cannot be fully explained by selectivity for spatial structure captured by the gist model or selectivity for low-level visual features captured by the motion-energy model. $c$, The proportion of variance explained commonly by category/gist $(C \cap G)$, category/motionenergy $(C \cap M)$, and gist/motion-energy $(G \cap M)$ models in each ROI (mean \pm SEM category selectivity onto flattened cortical surfaces. The surfaces were reconstructed in each individual subject from $\mathrm{T}_{1}$-weighted brain scans. These anatomical data were processed in Caret for gray-white matter segmentation (Van Essen et al., 2001). Surfaces were constructed from the segmentations separately for each hemisphere. The cortical surfaces were then flattened after applying five relaxation cuts placed so as to minimize spatial distortion. To project voxelwise category models onto the generated flat maps, functional data were aligned to the anatomical data using in-house Matlab scripts (MathWorks). These scripts used affine transformations to manually coregister three-dimensional functional and anatomical datasets (Hansen et al., 2007).

Spatial segregation of voxel clusters. The cluster analysis procedure described above was applied to voxelwise tuning profiles without including any information about the spatial location of the voxels. Thus, that analysis alone does not provide any information about whether clusters identified within scene-selective ROIs are spatially segregated in the cortex. If clusters are segregated spatially, then the three-dimensional anatomical distances among voxels within each cluster should be smaller than the distances among voxels between different clusters. In contrast, if clusters are intermingled, within-cluster and between-cluster distances should be similar. Therefore, to determine whether functionally distinct clusters are also clustered anatomically, we first measured the three-dimensional anatomical distance between every pair of voxels within each individual brain, and we then aggregated these distances within and between clusters separately. We used bootstrap tests to compare these distances to null distributions of within-cluster and between-cluster distances obtained by randomly shuffling the anatomical locations voxels in each individual ROI and in each individual brain.

\section{Results}

Representation of nonscene categories in the PPA, RSC, and OPA

There is substantial evidence that the three scene-selective areas examined here-the PPA, RSC, and OPA-represent information about natural visual scenes (Grill-Spector and Malach, 2004; Spiridon et al., 2006; Nasr et al., 2011). However, these areas also appear to represent information about nonscene categories (Huth et al., 2012; Stansbury et al., 2013), though this is poorly understood. Because natural scenes contain many distinct objects and actions, elucidating the representations of objects and actions in scenes is a challenging problem. To investigate this issue, we assessed selectivity for hundreds of object and action categories in the PPA, in the RSC, and in the OPA. We recorded BOLD responses from six subjects who viewed $2 \mathrm{~h}$ of natural movies, and we fit category models to each individual voxel in every subject. This enabled us to estimate voxelwise selectivity for 1705 separate object and action categories (Fig. 1). We find that the category model yields significant prediction scores in all ROIs: $0.38 \pm 0.08$ in the PPA (correlation; mean \pm SD across subjects), $0.38 \pm 0.09$ in the RSC, and $0.40 \pm 0.06$ in the OPA. All these values are statistically significant $\left(p<10^{-4}\right.$, bootstrap test). As a control, we fit separate gist models that reflect voxelwise selectivity for the spatial texture and layout of visual scenes. The gist model also yields significant prediction scores: $0.12 \pm 0.03$ in the PPA, $0.11 \pm 0.08$ in the RSC, and $0.11 \pm 0.03$ in the OPA $(p<$ $\left.10^{-4}\right)$. However, the category model performs significantly better than the gist model in all three ROIs $\left(p<10^{-4}\right)$. These results indicate that voxel responses in scene-selective areas carry significant information about object and action categories in natural scenes.

across subjects). A relatively small portion of variance is explained jointly by category and motion-energy models $\left(p<10^{-4}\right)$. Therefore, to reduce spurious correlations, a nuisance motion-energy regressor was included in the category models during subsequent analyses. 
While early visual areas are commonly thought to represent low-level stimulus features (Grill-Spector and Malach, 2004; Kay et al., 2008), recent studies suggest that downstream scene-selective areas might represent both low-level features (Rajimehr et al., 2011) and global spatial structure (Walther et al., 2009, 2011; Kravitz et al., 2011b). Because objects and actions in natural movies are partly correlated with low-level features, the category models estimated in scene-selective ROIs might be biased. Thus we sought to determine whether the category model still explains a significant portion of the response variance in the PPA, RSC, and OPA, after accounting for variance that can be attributed to low-level features or scene structure. We used a variance partitioning analysis to address this issue (Fig. $2 a$; see Materials and Methods). The variance partitioning analysis included three separate models: the category and gist models discussed above and a separate motionenergy model that characterizes voxel selectivity for low-level structural features, including spatial position, spatiotemporal frequency, and orientation. We calculated the proportion of shared variance explained by multiple models and the proportion of variance explained uniquely by each model.

We performed the variance partitioning analysis for each of our subjects individually, focusing on retinotopically organized early visual areas (V1-V3) and the PPA, RSC, and OPA (Fig. 2b). If the category model explains a portion of the response variance that cannot be attributed to the motion-energy or gist models, then addition of the category model regressors should improve the total explained variance. We find that the percentage of explained variance that can be attributed uniquely to the category model is $39.4 \pm 7.3 \%$ (mean $\pm \mathrm{SD}$ across subjects) in the PPA, $43.8 \pm 11.0 \%$ in the RSC, and $39.2 \pm 6.7 \%$ in the OPA $(p<$ $10^{-4}$, bootstrap test), but it is insignificant in retinotopic areas $(p>0.3)$. This result suggests that scene-selective areas represent significant information about object and action categories in natural scenes. Importantly, the variance partitioning procedure ensures that this information cannot be attributed to selectivity for low-level features as reflected in the gist or motion-energy models. At the same time a relatively small portion of variance is explained commonly by category and motionenergy models ( $p<10^{-4}$; Fig. $2 c$ ). Therefore, to reduce spurious correlations in subsequent analyses presented in this paper, a nuisance motion-energy regressor was included in the category models (see Materials and Methods).

\section{Stability CDFs}
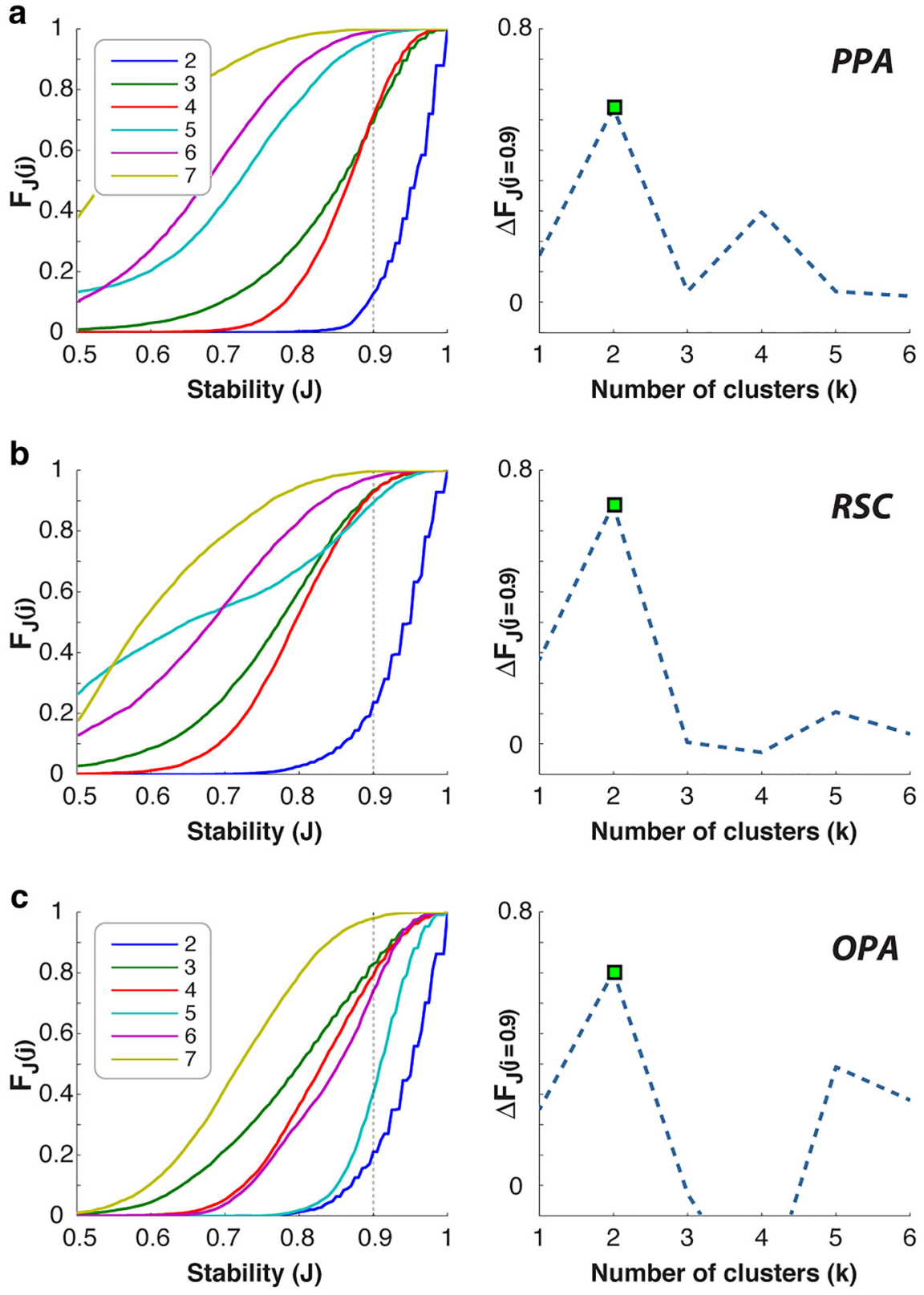

Figure 3. The optimal number of clusters for each scene-selective ROI. An unsupervised stability-based validation technique was used to determine the optimal number of clusters in three scene-selective ROls. $\boldsymbol{a}$, Cluster analysis for the PPA. Left, The cumulative distribution function of clustering stability, $F_{J}(j)$, shown as a function of number of clusters $(k)$ ranging from 2 to 7. Right, Change in value of $F_{j}$ across consecutive $k$ at a stability threshold of $J=0.9$ (Ben-Hur et al., 2002). The optimal $k$ was identified by detecting a sudden transition from narrow to widespread distributions. This transition was identified by a large increase in the value of $F$, when gradually increasing the number of clusters. The optimum number of clusters in the PPA is two (data are aggregated across subjects and hemispheres). $\boldsymbol{b}$, Cluster analysis for the RSC. Format same as in $\boldsymbol{a}$. The optimum number of clusters in the RSC is two. c, Cluster analysis for the OPA. Format same as in $\boldsymbol{a}$. The optimum number of clusters in the OPA is two.

\section{Functional heterogeneity in the PPA, RSC, and OPA}

Several recent studies report that subregions within the PPA vary in their visual responsiveness and spatial-frequency tuning (Arcaro et al., 2009; Rajimehr et al., 2011; Baldassano et al., 2013). These findings suggest that the PPA, and perhaps other sceneselective ROIs, might contain multiple subdivisions with different category selectivity. To test this heterogeneity hypothesis, we first sought to determine whether individual voxels in the PPA, RSC, and OPA differ in their tuning for object and action catego- 
Table 1. Distribution of voxels across clusters identified in scene-selective ROIs

\begin{tabular}{lrrrrrr}
\hline & PPA1 & PPA2 & RSC1 & RSC2 & OPA1 & OPA2 \\
\hline Total & 277 & 243 & 185 & 228 & 223 & 184 \\
S1 & 11 & 88 & 16 & 54 & 4 & 49 \\
S2 & 36 & 11 & 13 & 19 & 7 & 6 \\
S3 & 39 & 34 & 52 & 66 & 62 & 46 \\
S4 & 59 & 28 & 13 & 22 & 68 & 30 \\
S5 & 91 & 73 & 19 & 49 & 38 & 42 \\
S6 & 41 & 9 & 72 & 18 & 44 & 11 \\
\hline
\end{tabular}

The first row shows the total number of voxels within each cluster, pooled across subjects. Subsequent rows show the number of voxels within each cluster in individual subjects $(S 1-S 6)$.

ries. We reasoned that if model weights are significantly different across voxels within an ROI, then the category model fit to an individual voxel (i.e., self-prediction) should explain more of that voxel's response than can be explained using category models fit to other voxels (i.e., cross-prediction). Comparison of the selfprediction and cross-prediction performance of category models in all voxels within each ROI shows that self-prediction improves explained variance by $24.1 \pm 9.5 \%$ in the PPA (mean \pm SD across subjects), by $28.5 \pm 11.0 \%$ in the RSC, and by $25.0 \pm 15.4 \%$ in the OPA $\left(p<10^{-4}\right.$, bootstrap test). These results confirm that voxels within the PPA, RSC, and OPA are functionally heterogeneous.

We next tested whether the heterogeneously tuned voxels in scene-selective ROIs form distinct functional clusters. To do this, we first applied spectral clustering to the voxelwise category model weights obtained within each area. We performed a stability-based validation procedure to determine the optimal number of clusters in the PPA, RSC, and OPA separately, and we measured cluster stability by repeating the cluster analysis 5000 times on subsets of voxels selected randomly in each random draw (see Materials and Methods). We find that in all three ROIs, the optimal number of clusters based on the category model is two (Fig. 3; for voxel numbers across clusters, see Table 1). To determine whether these clusters are consistent across subjects, we measured the intersubject correlation of cluster centers, where the cluster center was taken as the average model weight within a cluster. We find that individual-subject clusters are highly consistent across subjects $(r=0.84 \pm 0.04$ in the PPA, $0.81 \pm 0.03$ in the RSC, and $0.72 \pm 0.04$ in the OPA; mean \pm SD across subjects, $p<10^{-4}$, bootstrap test), and that they are consistent with the group clusters $(0.92 \pm 0.03$ in the PPA, $0.91 \pm 0.02$ in the RSC, and $0.87 \pm 0.04$ in the OPA, $\left.p<10^{-4}\right)$. For comparison, we also performed the same cluster analysis procedure separately using the gist model and the motion-energy model. In all three ROIs, the optimal number of clusters based on the gist and motion-energy models is one. Together, these results confirm that voxels within the PPA, RSC, and OPA are functionally clustered according to their category selectivity, but they are not clustered for lower-level features.

We next examined whether the differential category selectivity between these two voxel clusters are functionally important. We reasoned that if the intercluster differences are important, a target voxel's responses should be better explained by models fit to other voxels in the same cluster (within-cluster prediction) than by models fit to voxels in a different cluster (cross-cluster prediction). Therefore, we simply compared the within-cluster and cross-cluster prediction performance in the PPA, RSC, and OPA. Separate response predictions were obtained using category, gist, and motion-energy models (Fig. 4). We find that within-cluster performances based on category models are significantly higher than cross-cluster performances in all three ROIs $(p<0.001$, bootstrap test). For category models, percentage improvement in

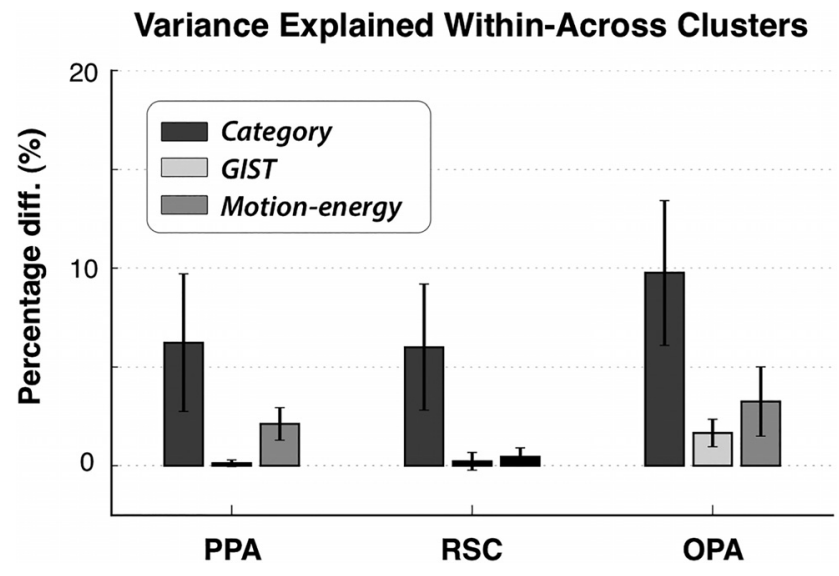

Figure 4. Functional segregation of subdomains. If subdomains identified within an ROI are functionally distinct, then the variance of a voxel's response explained by other voxels in the same subdomain (within-subdomain prediction) should be greater than the variance explained by voxels in different subdomains (cross-subdomain prediction). We therefore compared within-prediction and cross-prediction performances based on responses predicted by the category, gist, and motion-energy models. Bar plots show the percentage difference in explained variance in the PPA, RSC, and OPA (mean \pm SEM across subjects). Greater percentages indicate better within-prediction than cross-prediction performance. Insignificant differences are shown in blank outlines $(p>0.05)$. In all ROls, the within-prediction performance of the category model is greater than the cross-prediction performance ( $p<0.001$, bootstrap test). This result suggests that there are functional subdomains with distinct category tuning in the PPA, RSC, and OPA.

explained variance is $6.3 \pm 3.6 \%$ (mean \pm SEM across subjects) in the PPA, $6.0 \pm 3.2 \%$ in the RSC, and $9.6 \pm 3.6 \%$ in the OPA. These results strongly support the hypothesis that there are two functional subdomains with distinct category tuning in the PPA, RSC, and OPA.

To examine the differences in category tuning between these subdomains, we first visualized the cluster center weights for 1705 categories in each scene-selective ROI (Fig. 5 for group centers; see Figs. 12-14 for individual-subject centers). Figure 6 summarizes the responses of each cluster to several important object and action categories, along with response differences between the two clusters. BOLD responses of both the first and second cluster in each ROI (here denoted as PPA1, RSC1, and OPA1 for cluster 1, and PPA2, RSC2, and OPA2 for cluster 2) increase when structures, man-made instruments, vehicles, and movement are present ( $p<0.001$, bootstrap test). Responses of these same clusters are reduced by scenes presenting social communication, such as people talking or gesturing $(p<0.001)$. Furthermore, both clusters yield greater responses for man-made instruments and vehicles than for buildings and geological formations $(p<$ 0.001 ). However, in every ROI the two functional clusters differ in their relative responses to these categories and to other ecologically relevant categories. Specifically, the first cluster (PPA1, $\mathrm{RSC} 1$, and OPA1) produces relatively greater responses than the second cluster when natural materials, body parts, humans, animals, and social communication are present in the movies $(p<$ $0.05)$. In contrast, the first cluster produces relatively reduced responses when the movies show movement, such as a moving car or train, or a walking person $(p<0.001)$. Furthermore, responses in the RSC1 and OPA1 are reduced when vehicles are present $(p<0.001)$. These results suggest that the first subdomain in scene-selective areas has stronger tuning for animate objects and man-made instruments, while the second subdomain is relatively more tuned for vehicles and action categories that appear in dynamic visual scenes. 

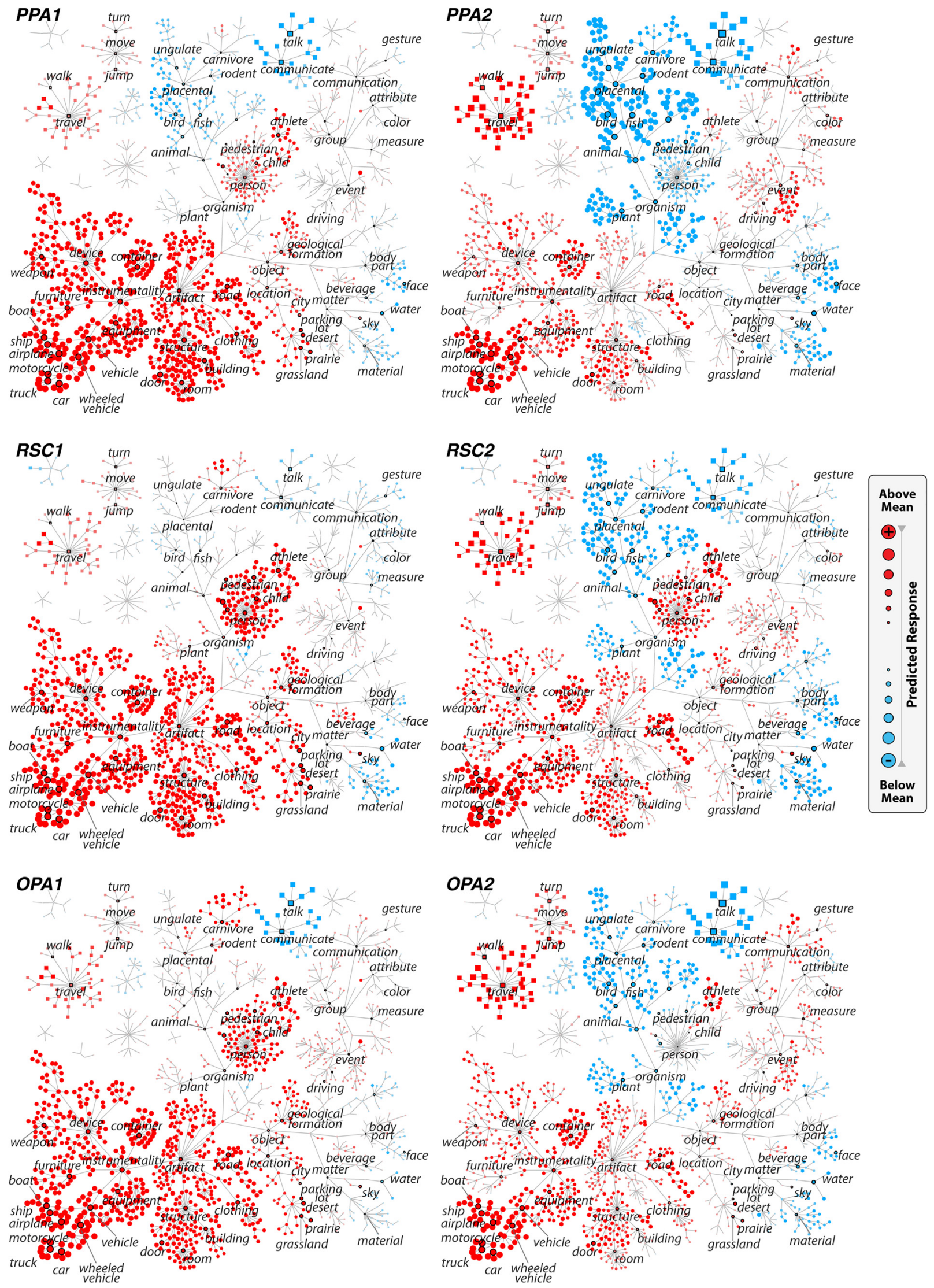

Figure 5. Category tuning of the voxel clusters. Category tuning of the two functional subdomains identified in each scene-selective R0I. For each cluster, category tuning was taken as the mean tuning profile of all voxels within the ROI (data are aggregated across subjects and hemispheres). Tuning for 1705 categories are shown here using graphs that consist of separate trees for object (main tree, circular vertices) and action (smaller trees, square vertices) categories. To orient the reader, a subset of the categories has been labeled. The size of each vertex indicates the magnitude while its color indicates the sign (red, +; blue, -) of the category response relative to the mean overall response. Left, Responses of PPA1, RSC1, and OPA1 are (Figure legend continues.) 

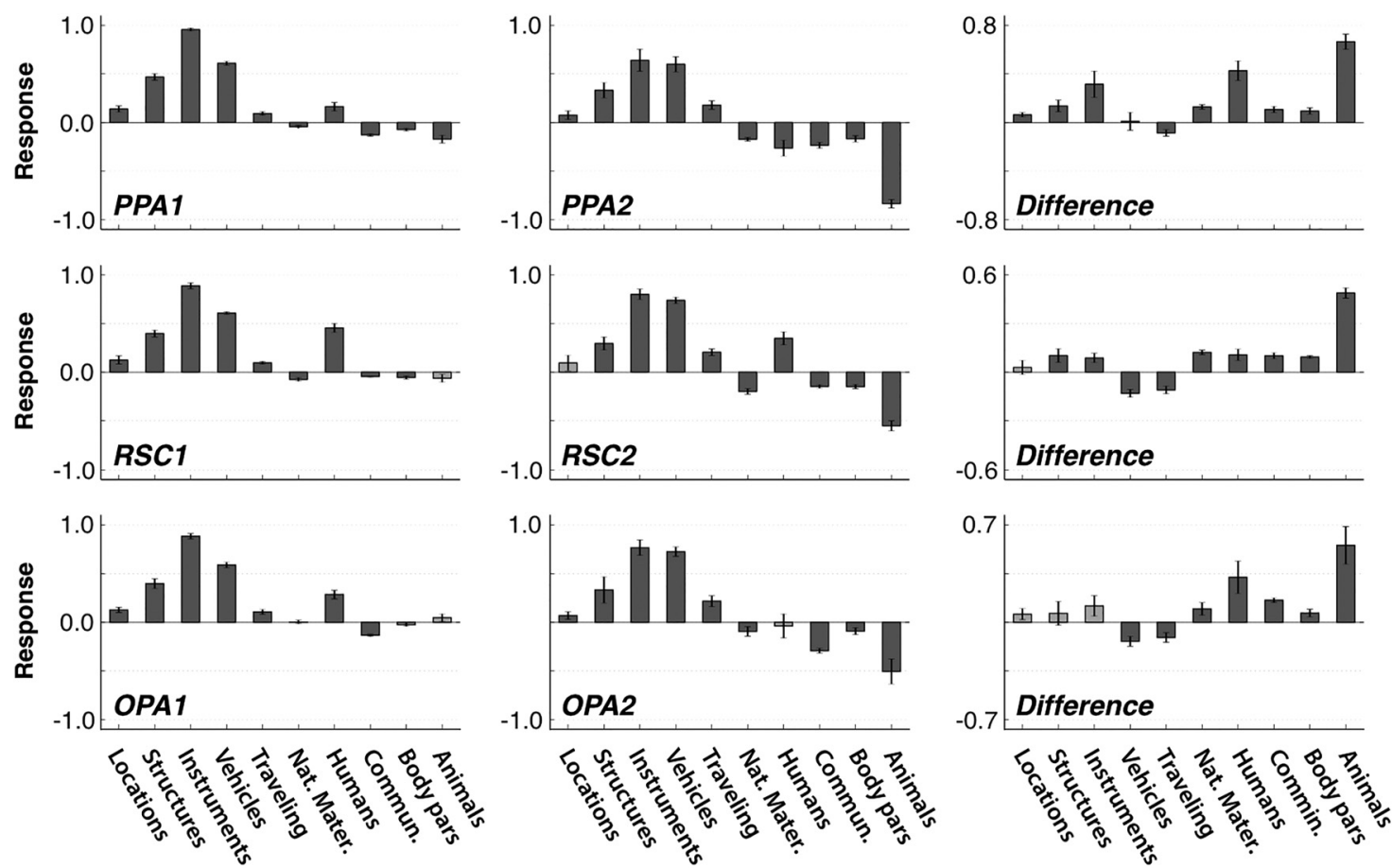

Figure 6. Predicted differences in category responses across clusters. The voxelwise category models fit to all voxels within each subdomain were used to estimate predicted responses to geographic locations, structures (e.g., building), instruments, vehicles, movement, natural materials, humans, social communication, body parts, and animals. The response level for each of these superordinate categories was taken as the average response across all of its subordinate categories included in the category model. Bar plots show the response level (mean \pm SEM across subjects) for the two subdomains in each ROI as well as their difference (right column). Significant responses are shown in dark gray ( $p<0.05$, bootstrap test) and insignificant responses are shown in light gray. Relative to the second subdomain, the first subdomain is observed to respond less to traveling, and relatively more to most of the remaining categories including those related to humans, communication, and structures.

Several recent studies have reported variability in visual selectivity across the anterior-posterior axis of the PPA that also extends into neighboring patches of the cortex (Arcaro et al., 2009; Rajimehr et al., 2011; Baldassano et al., 2013). It is therefore possible that category tuning follows a similar organization within and nearby the three scene-selective areas examined here. Alternatively, voxel clusters may show a patchy, noncontiguous spatial distribution (Grill-Spector et al., 2006). To examine this issue, we measured the category tuning profiles of all voxels within a $40 \mathrm{~mm}$ radius of the geometric center of each sceneselective ROI in each hemisphere. Separate principal component (PC) analyses on category tuning profiles of voxels located within each cluster reveal that the two clusters are clearly distinguished by the first PC in each of the three ROIs (see below, PC analyses of category models). To visualize these patterns, we mapped the first PC projections of 1705-dimensional tuning profiles onto the cortical surface. In Figure 7, voxels that belong to PPA1, RSC1, and OPA1 have positive projections onto the first PC, while voxels in PPA2, RSC2, and OPA2 have negative projections. Inspection of these projections on cortical flatmaps suggests that voxels in the first cluster tend to be located approximately in posterior-lateral regions, and voxels in the second cluster tend to be located more anteriomedially. Supporting this observation, a statistical analysis indicates that there is significant spatial segregation between

$\leftarrow$

(Figure legend continued.) strongly increased by the presence of man-made instruments, devices, vehicles, structures, roads, and locations (e.g., city, grassland), and they are weakly increased by the presence of humans. Right, Responses of PPA2, RSC2, and OPA2 are strongly increased by the presence of vehicles, roads, and traveling, and they are strongly reduced by animals, plants, natural materials, body parts, and communication. the two clusters $(p<0.01$, bootstrap test; see Materials and Methods). On the other hand, this segregation is not complete and some degree of intermixing between the clusters appears to occur within each ROI. Together, these results imply that category representation across the PPA, RSC, and OPA are likely organized by both monotonic gradients and distributed peaks of selectivity.

\section{PC analyses of category models}

Evidence from recent studies suggests that the human brain embeds visual categories into a relatively low-dimensional semantic space mapped systematically across the cortical surface (Haxby et al., 2011; Huth et al., 2012). To obtain a data-driven description of the semantic information represented in scene-selective areas, we performed PC analyses across voxelwise category models. We assessed the consistency of representations across subjects by evaluating the cross-subject correlations between the PCs estimated for individual subjects (Huth et al., 2012). To avoid stimulus-sampling bias, we measured correlations between PCs that were estimated separately from responses to the first and second halves of the movies. We find that the first three individual-subject PCs are highly correlated across subjects $(r=$ $0.61 \pm 0.02$ in the PPA, $0.54 \pm 0.01$ in the RSC, and $0.57 \pm 0.01$ in the OPA; mean \pm SD across subjects, $p<10^{-4}$, bootstrap test). These individual-subject PCs are also highly correlated with the group PCs in all three areas $(0.71 \pm 0.03$ in the PPA, $0.62 \pm$ 0.01 in the RSC, and $0.64 \pm 0.02$ in the OPA, $p<10^{-4}$ ).

Our cluster analyses indicate that voxels in each of the three ROIs form two clusters that differ in their category tuning. To examine the semantic dimensions that capture these tuning differences, we projected the voxelwise tuning profiles onto the first 

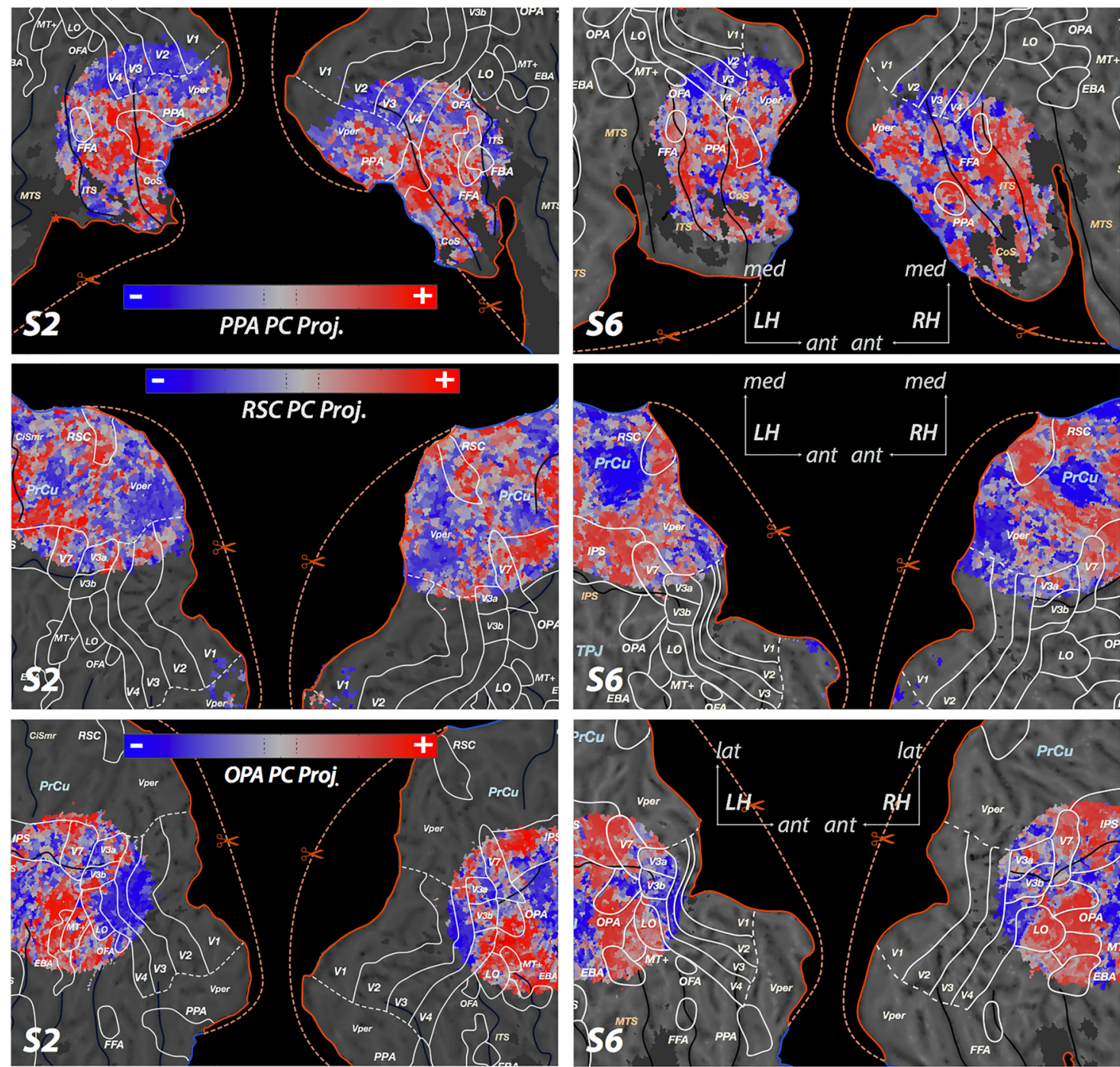

Figure 7. Cortical flatmaps of category selectivity within and outside scene-selective ROls. To examine the spatial distribution of category selectivity, category tuning profiles were measured for voxels within and around the PPA, RSC, and OPA separately. The tuning profiles in the vicinity of each ROI were then projected onto the first group PC (calculated only from voxels within the given ROI). Here the projections obtained for the PPA, RSC, and OPA are shown on separate cortical flatmaps for two representative subjects S2 and S6. Brain areas identified using functional localizers are labeled and their extent is delineated with white lines. Voxels with positive projections onto the PC (i.e., category tuning more similar to PPA1, $\mathrm{RSC}$, and OPA1) appear in red, and voxels with negative projections (i.e., category tuning more similar to PPA2, RSC2 and OPA2 appear in blue. The two voxel clusters in each ROI show spatial segregation on the cortical surface.

\section{PC Projections}
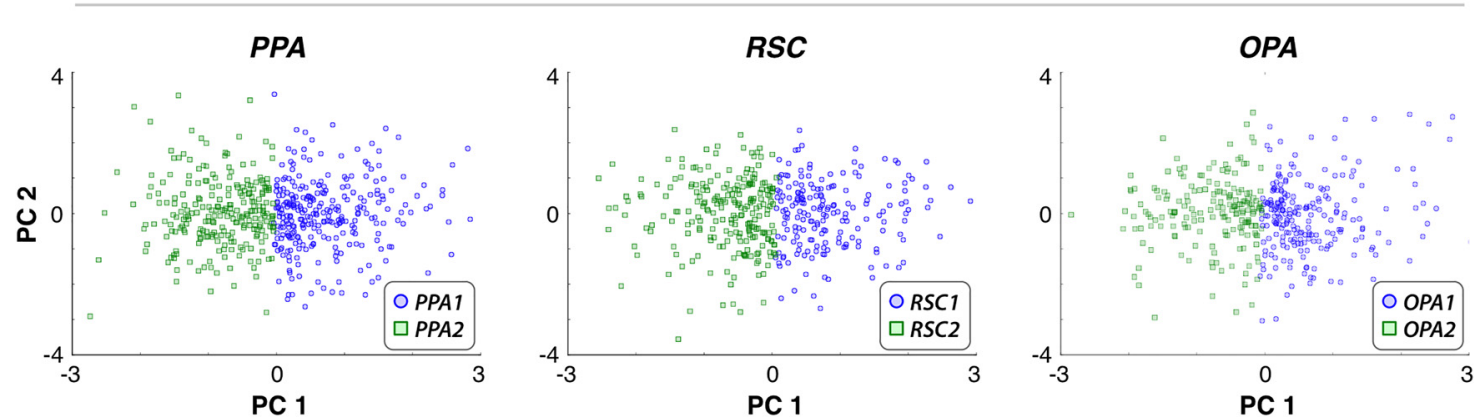

Figure 8. Projections of voxelwise tuning profiles onto PCs. To independently assess the functional heterogeneity in scene-selective ROIs, voxelwise tuning profiles were projected onto the first two group PCs obtained from voxels within each ROI (data aggregated across subjects and hemispheres). Each voxel in the first cluster (PPA1, RSC1, and OPA1) is denoted with a blue circle, and each voxel in the second cluster (PPA2, RSC2, and OPA2) is denoted with a green square. Voxels in separate clusters are spatially segregated in the PC space. Projections onto the first PC clearly separate voxels in the first and second clusters, implying that category representation in scene-selective Rols is organized according to at least one semantic dimension. 


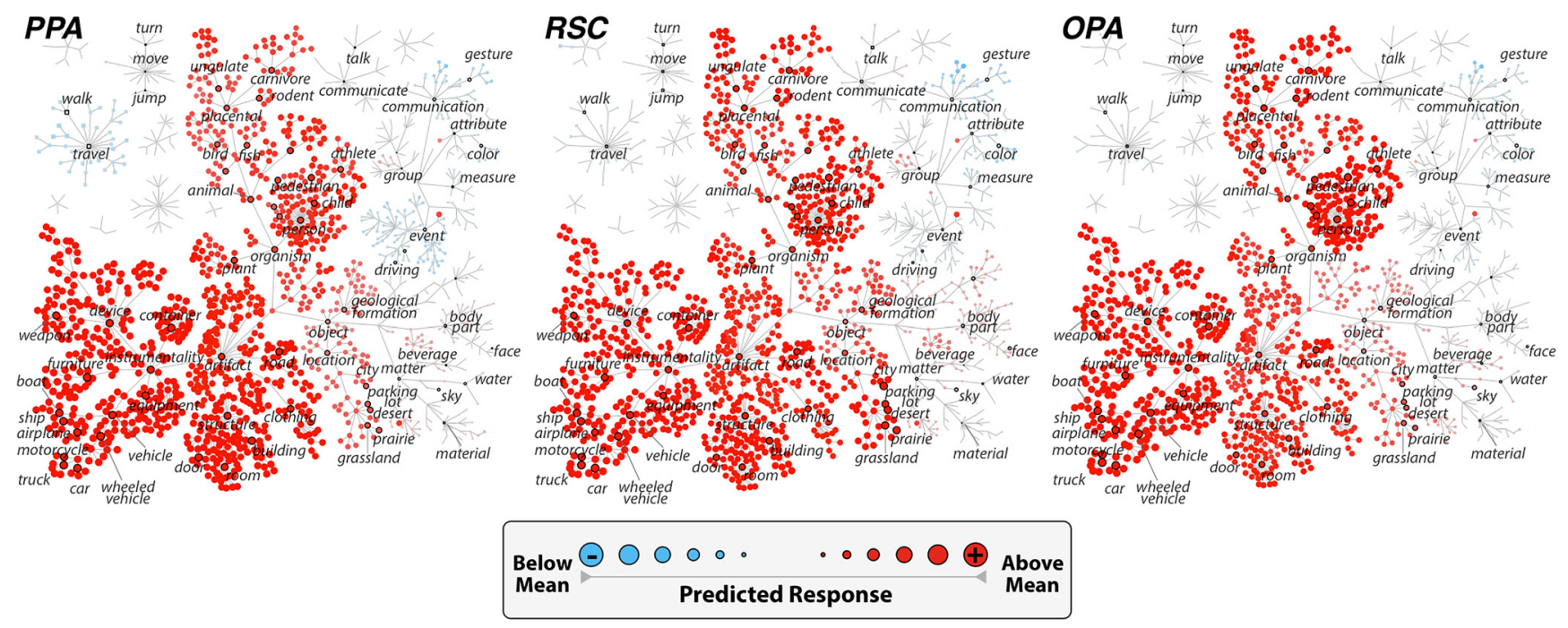

Figure 9. Group PCs of tuning profiles in scene-selective ROIs. PC weights for the PPA, RSC, and OPA. Tuning diagrams are formatted as in Figure 5. Inspection of the tuning diagrams of the first $\mathrm{PC}$ reveals the semantic dimension that distinguishes the two subdomains in each scene-selective ROI. The first PC approximately contrasts categories related to human civilization and man-made artifacts (e.g., instruments, vehicles, roads, indoor spaces, and humans) with categories related to social interaction (e.g., communication) and outdoor activities (e.g., outdoor events, movement, and natural materials). Thus the first subdomain tuned for objects commonly appearing in static scenes has positive projections onto this $\mathrm{PC}$, while the second subdomain tuned for object and actions in dynamic scenes has negative projections.

two group PCs of category models in each area. Across subjects, the first and second PCs explain $48.4 \pm 10.8 \%$ and $15.1 \pm 2.6 \%$ of category responses in the PPA, $48.1 \pm 8.9 \%$ and $15.7 \pm 4.3 \%$ in the RSC, and $58.9 \pm 9.1 \%$ and $14.2 \pm 5.7 \%$ in the OPA. This result indicates that voxels in separate clusters project to segregated regions in the semantic space defined by the selected PCs (Fig. 8). Inspection of Figure 8 reveals that the first PC clearly captures the differences in category tuning between the two clusters in the PPA, RSC, and OPA. As shown in Figure 9, this first PC appears to contrast categories related to civilization (e.g., instruments, vehicles, roads, indoor spaces, and humans) with categories related to social interaction (e.g., communication) and outdoor activities (e.g., outdoor events, movement, and natural materials). While a more precise interpretation of PCs across a 1705-dimensional feature space is naturally difficult, our results suggest that category representation is organized consistently across subjects according to at least one semantic dimension.

\section{Hemispheric symmetry of category representations}

Several previous studies suggest that brain function in categoryselective areas in the high-level visual cortex are lateralized across hemispheres (Rossion et al., 2000; Stevens et al., 2012). We therefore asked whether the voxel clusters identified in the PPA, RSC, or OPA are lateralized. To address this issue, we first counted the number of voxels included in the definition of scene-selective areas in the left and the right hemispheres separately. We find no consistent hemispheric lateralization in ROI definitions across subjects for the PPA ( $p>0.15$, bootstrap test). However, $73.7 \pm$ $11.4 \%$ of all RSC voxels and $66.4 \pm 26.9 \%$ of all OPA voxels (mean \pm SD across subjects) are located in the right hemisphere $(p<0.05)$. We next examined the distribution of voxels across the two hemispheres for individual clusters (subjects S1 and S2 had no OPA voxels in the left hemisphere and so were omitted from this analysis). For each cluster, we computed the ratio of the voxels in a given hemisphere to the total number of voxels across both hemispheres. We find that there is no significant lateralization for either of the two clusters in the PPA, RSC, or OPA ( $p>$ 0.30 , bootstrap test). This result indicates that subdomains in scene-selective areas are relatively balanced across cerebral hemispheres.

\section{Control analyses for potential confounds caused by bias in the movie stimulus}

We report here that the mean category tuning profiles of the voxel clusters are highly consistent across individual subjects in the PPA, RSC, and OPA. However, we were concerned that these results might be an artifact of statistical bias in the natural movies used as stimuli in the main experiment. After all, voxelwise tuning profiles were measured using responses elicited by the same stimulus in all subjects. Any natural stimulus of finite duration will inevitably reflect some degree of stimulus sampling bias and, if this bias is significant, then it might increase the apparent similarity of model weights calculated across subjects. To rule out this potential bias, we fit separate models to responses recorded during the first and second halves of the movie. The clips used in the first and second halves of the movie were completely unrelated, so if the results are consistent across the two halves then it would suggest that statistical bias in the movies is not an important concern. We ran cluster analyses individually on each set of models, and we compared the resulting cluster centers. We find that the split-half cluster centers are strongly correlated across subjects $(r=0.79 \pm 0.04$ in the PPA, $0.75 \pm 0.01$ in the RSC, and $0.60 \pm 0.07$ in the OPA, mean $\pm \mathrm{SD}, p<10^{-4}$, bootstrap test). This result indicates that the consistency of clusters across subjects is unlikely to be due to stimulus sampling bias.

Another potential confound stems from the correlations among different categories in the finite movie stimulus used in this study. Multiple distinct categories of objects and actions may co-occur in natural movies. If these category correlations are large, then the corresponding category regressors used in our voxelwise models will be highly correlated, which might bias the fit model weights. To assess the effect of category correlations on model fits, we measured the amount of variance in the voxelwise category model weights that can be attributed to the stimulus time course. To account for temporally lagged correlations, we concatenated multiple delayed time courses for all 1705 catego- 

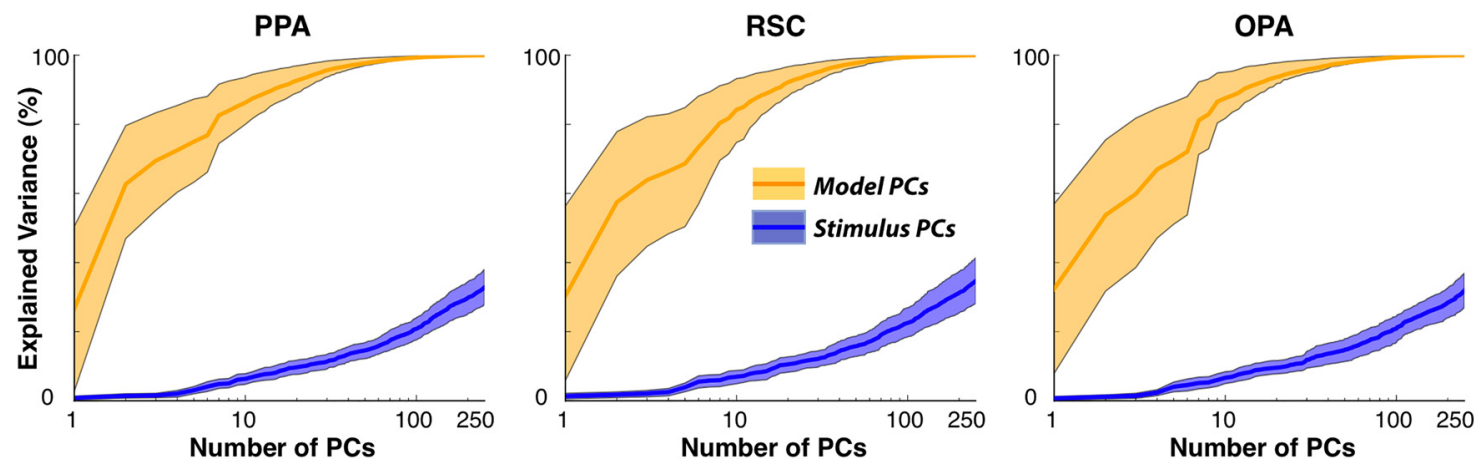

Figure 10. Temporal stimulus correlations between categories in natural movies. Regressors for multiple distinct categories of objects and actions may be correlated in the natural movie stimulus. To assess the effect of category correlations on our model fits, we compared the PCs of model weights with the PCs of the stimulus time course in terms of the amount of variance they can explain in voxelwise category models. Plots show the mean and 68th-percentile bands of the explained variance across the population of voxels in each ROI. Regardless of the number of PCs used, model PCs account for a significantly larger proportion of variance in the model weights compared with stimulus $\mathrm{PCs}\left(p<10^{-4}\right.$, bootstrap test). This result indicates that the estimated voxelwise category model weights are not biased by category correlations in the movie stimulus.

ries with lags ranging between -5 and $5 \mathrm{~s}$. We then calculated PCs of the resulting stimulus matrix, and separately calculated the PCs of the category model weights. If stimulus correlations strongly bias the model fits, then the stimulus PCs should explain a comparable portion of the variance in the model weights to that explained by the model PCs. We find that model PCs explain a significantly larger portion of the variance compared with the stimulus PCs (Fig. 10; $p<10^{-4}$, bootstrap test). In each ROI, we compared the combined explanatory power of all model PCs (total of 10 PCs) that individually explain $>1 \%$ of the variance in model weights with all stimulus PCs (total of 20 PCs) that each explain $>1 \%$ of variance in the stimulus matrix. We find that the variance in model weights explained by model PCs is $87.0 \pm 3.3 \%$ in the PPA (mean \pm SD across subjects), $83.9 \pm 3.2 \%$ in the RSC, and $87.6 \pm 3.0 \%$ in the OPA. In contrast, the variance in model weights explained by stimulus PCs was substantially smaller $\left(p<10^{-4}\right.$, bootstrap test $)$, merely $8.7 \pm 0.9 \%$ in the PPA, $9.9 \pm 0.8 \%$ in the RSC, and $9.1 \pm 1.2 \%$ in the OPA. This result indicates that the estimated voxelwise category model weights are not biased by category correlations in the movie stimulus.

One final potential confound concerns the correlation between low-level structural and high-level categorical features in natural scenes. If scene-selective areas represent low-level visual features (such as spatiotemporal frequency or orientation) that differ systematically across categories, then the category model weights might be biased. Of particular concern for this study is the possibility that the heterogeneity of category tuning across an area could reflect heterogeneity of tuning for

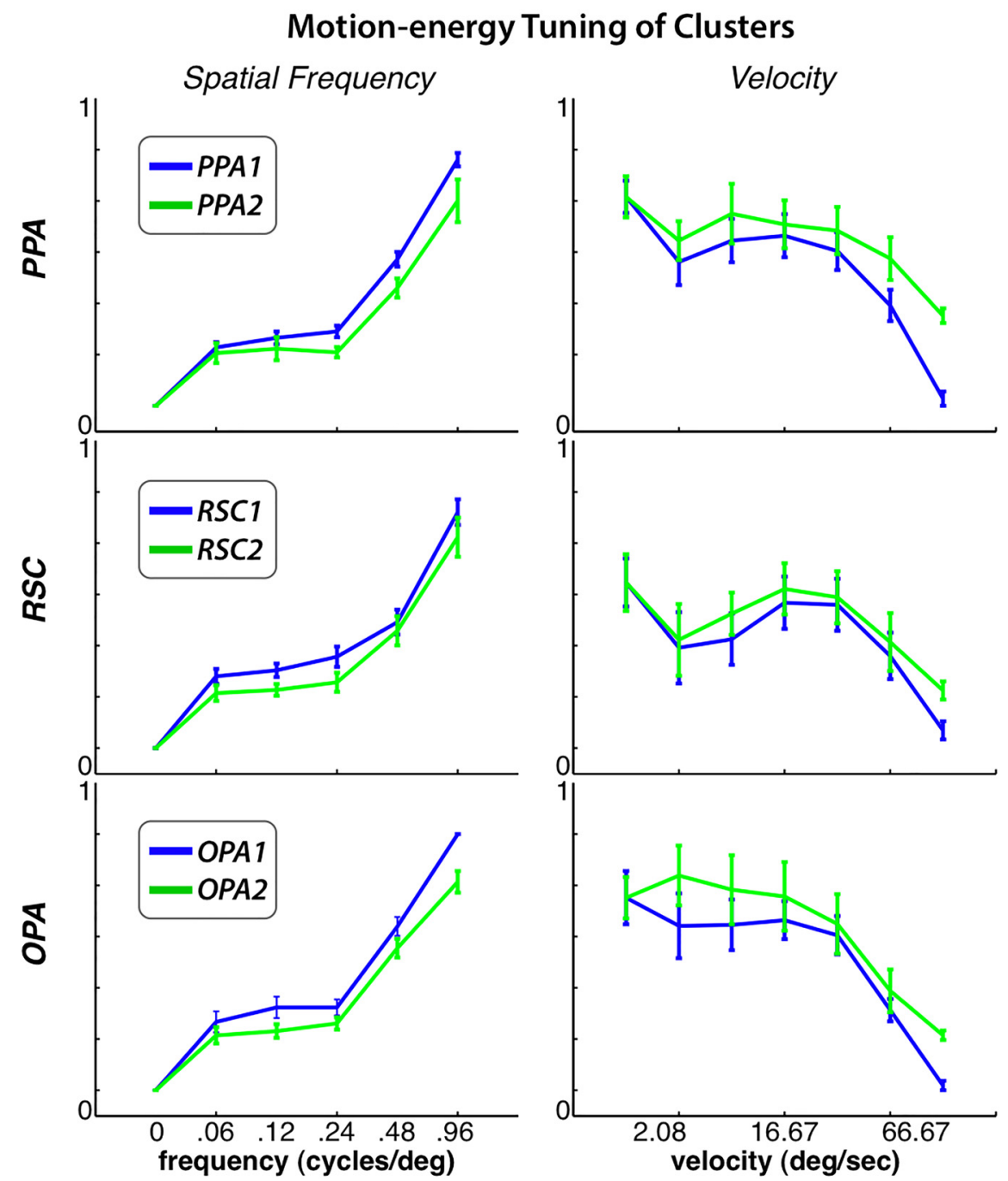

Figure 11. Motion-energy tuning of the voxel clusters. Differences in category tuning between the voxel clusters could potentially be confounded by differences in tuning for low-level structural features. To examine this issue, the mean motion-energy tuning of the clusters identified in Figure 5 were calculated for the PPA, RSC, and OPA separately. Spatial-frequency and velocity tuning profiles of the first (PPA1, RSC1, OPA1) and second (PPA2, RSC2, OPA2) voxel clusters are denoted with blue and green lines, respectively. Error bars indicate $S E M$ across voxels in each cluster. There are no significant differences in spatial frequency or velocity tuning of the two clusters in any of the three ROIs ( $p>0.05$, bootstrap test). This result suggests that differences in category tuning between the two subdomains identified in the PPA, RSC, and OPA cannot be attributed to heterogeneity of motion-energy tuning. 
PPA1
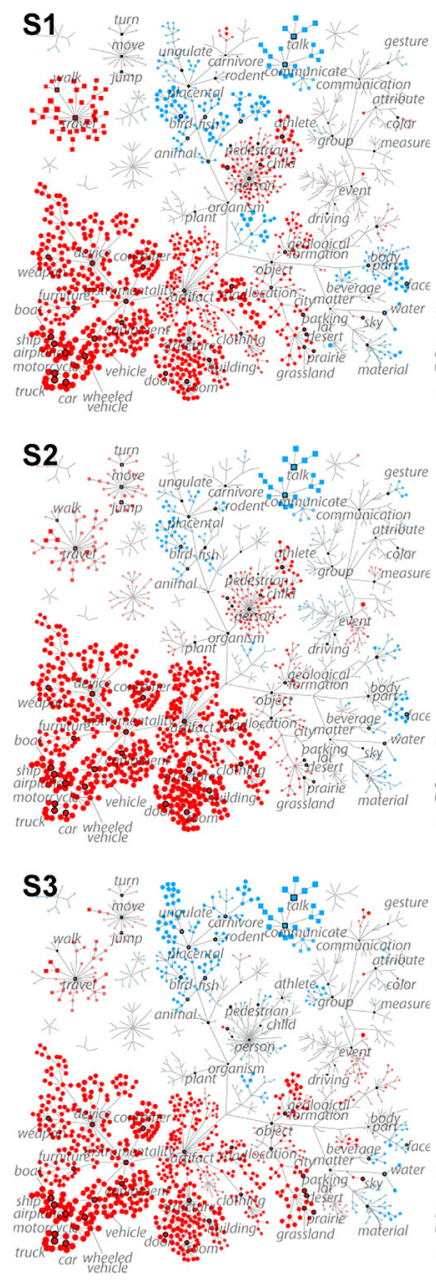

PPA2
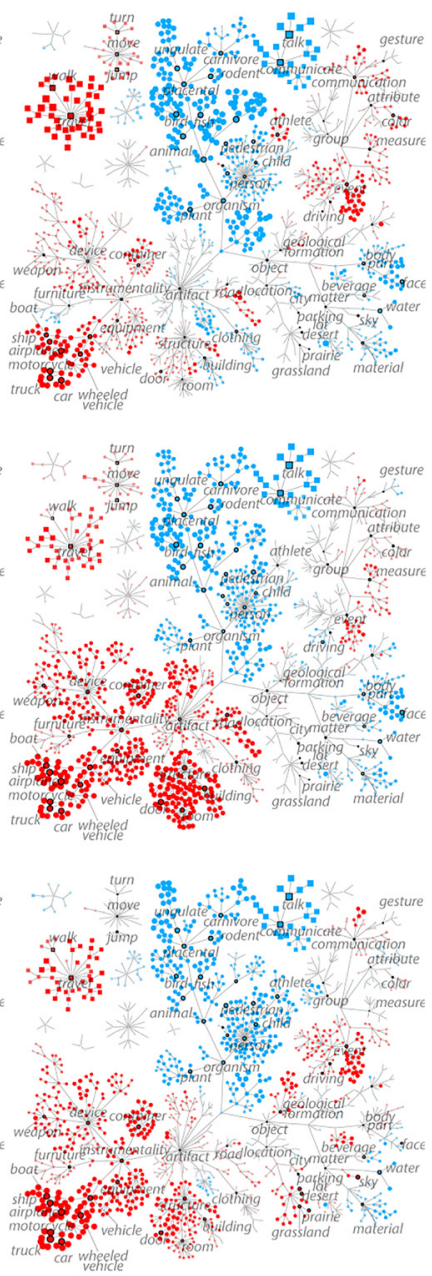

PPA1

PPA2
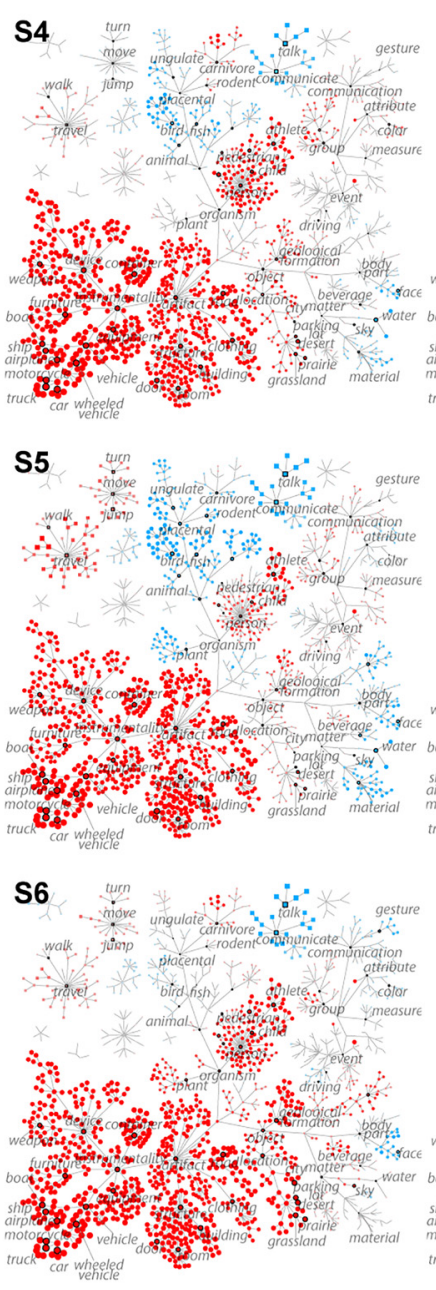
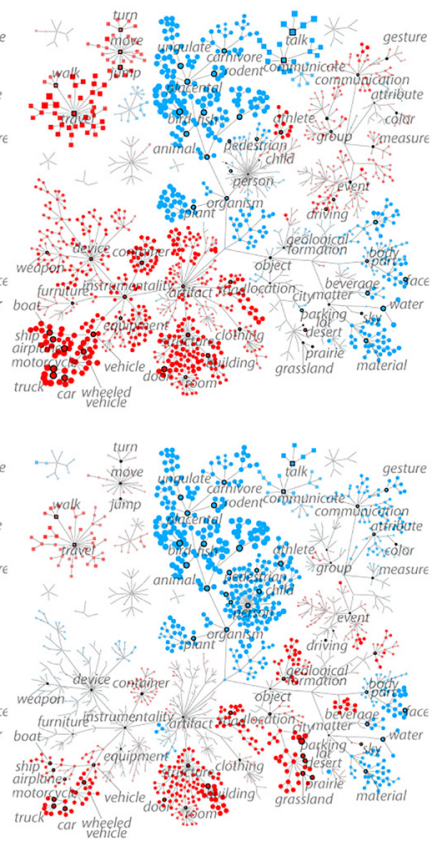

Figure 12. Individual-subject cluster centers in the PPA. Cluster analysis was performed separately in individual subjects (data are aggregated across hemispheres). The left and right panels for each subject show the centers of the two clusters in PPA1 and PPA2. The center of each cluster was taken as the mean tuning profile across voxels in that cluster. Tuning diagrams formatted as in Figure 5. The cluster centers are highly consistent across individual subjects.

low-level features. To examine this issue, we ran a control analysis to identify potential confounds due to correlated low-level features. First, we examined whether the two clusters obtained from category models in the PPA, RSC, and OPA show significant differences in motion-energy tuning. For this purpose, we fit voxelwise motion-energy models, and measured the mean motion-energy tuning across voxels in each of the clusters shown in Figure 5. The spatial frequency and velocity tuning of the two clusters identified in each ROI are displayed in Figure 11. We find no significant differences in motion-energy tuning of the voxel clusters in the PPA, RSC, and OPA ( $p>0.05$, bootstrap test). Together with the result described earlier showing that the motion-energy model produces only a single cluster in each of the three scene-selective ROIs, our data confirm that functional clustering of motion-energy tuning is much weaker than functional clustering of category tuning in the PPA, RSC, and OPA. Thus motion energy cannot explain the differences in category tuning between subdomains in these scene-selective areas.

\section{Discussion}

Scene-selective regions in the human visual cortex - the PPA, RSC, and OPA - are usually assumed to be functionally homogeneous. To determine whether this common assumption is true, we aimed to precisely map cortical function in these regions by leveraging the strength of fMRI in answering questions at the representational level. We used a voxelwise modeling framework to characterize the selectivity of single voxels in the PPA, RSC, and OPA to 1705 object and action categories in natural movies. As expected, we found that this model explains a greater portion of the response variance in the PPA, RSC, and OPA than is explained by a gist model based on spatial texture and layout. We then performed cluster analysis separately on the category models fit to voxels within each ROI, in each subject (Figs. 12-14). This analysis reveals two distinct functional subdomains in scene-selective ROIs. The first subdomain (PPA1, RSC1, and OPA1) is approximately located posteriolaterally and the second subdomain (PPA2, RSC2, and OPA2) is located anteriomedially within each area. While a definitive functional interpretation of these subdomains is not without challenge, our analyses reveal that the first subdomain yields relatively increased responses to man-made artifacts (buildings, furniture, devices, etc.), and that the second subdomain yields relatively increased responses to actions related to locomotion (e.g., walking, running, turning, or jumping) and to vehicles (e.g., car, boat, or bicycle). We find that these subdomains are highly consistent across all three sceneselective areas, across both hemispheres, and across individual 
RSC1
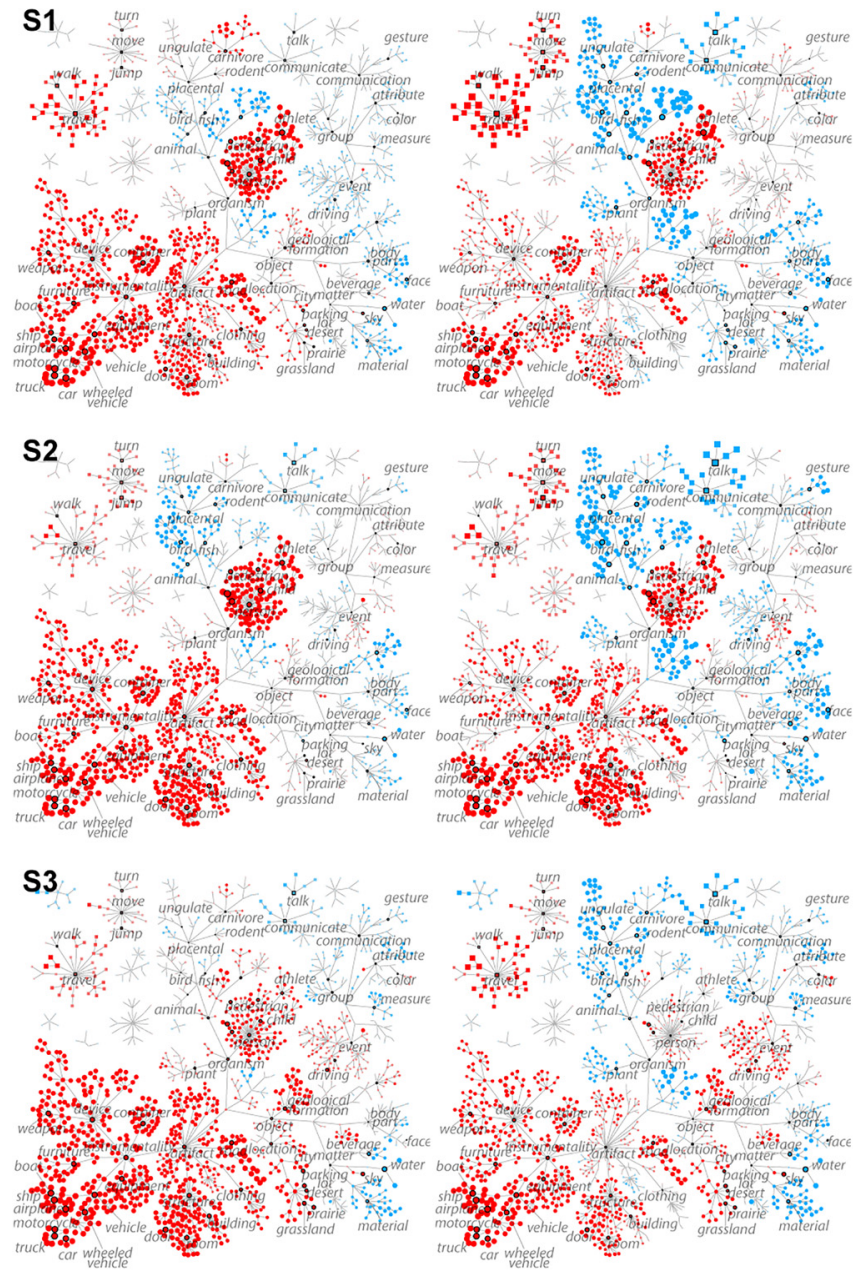

RSC2

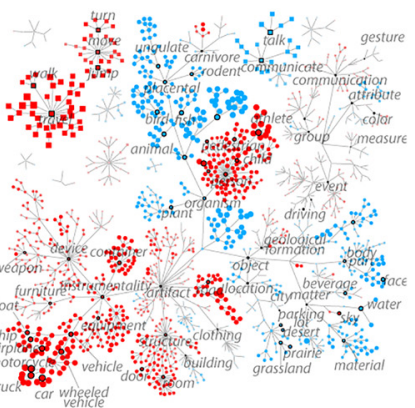

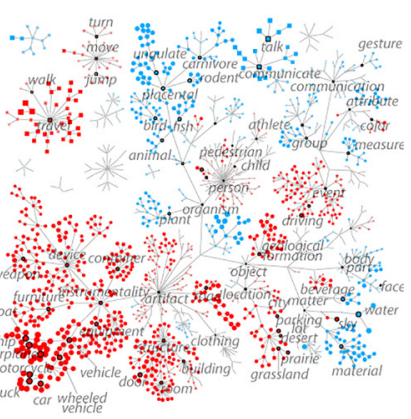

RSC1

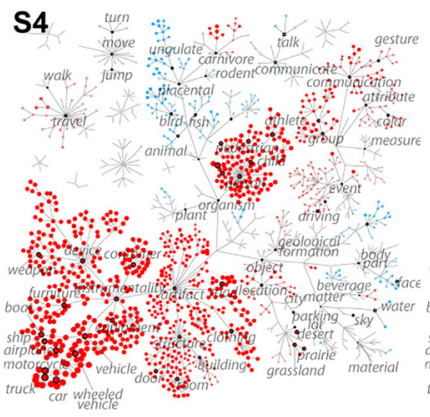

RSC2

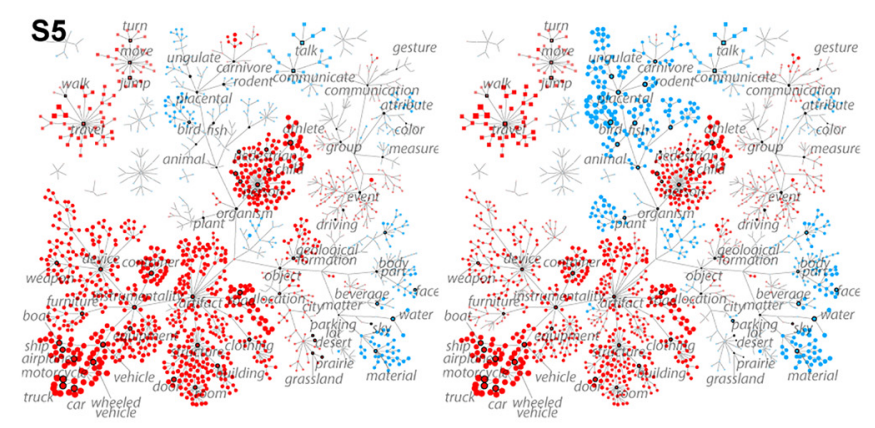

Figure 13. Individual-subject cluster centers in the RSC. Cluster analysis was performed separately in individual subjects (data are aggregated across hemispheres). The left and right panels for each subject show the centers of the two clusters in RSC1 and RSC2. The center of each cluster was taken as the mean tuning profile across voxels in that cluster. Tuning diagrams formatted as in Figure 5. The cluster centers are highly consistent across individual subjects.

subjects. One interesting possibility is that this consistent subdivision reflects an underlying anatomical organization of scene processing pathways into two processing streams, one biased toward static stimuli and one biased toward dynamic stimuli.

\section{Tuning for object and action categories in the PPA, RSC, and OPA}

In several previous reports, it has been questioned whether visual objects elicit significant responses in scene-selective ROIs, such as the PPA (Haxby et al., 2001; Downing et al., 2006; Reddy and Kanwisher, 2007; MacEvoy and Epstein, 2011). Based on BOLD responses elicited by isolated objects from a few different categories, such as houses, shoes, chairs, or cars, these earlier studies suggested that some inanimate object categories evoke weak (albeit significant) responses in the PPA. Several recent studies have further suggested that responses to nonscene objects in the PPA, RSC, and OPA depend critically on the object's navigational relevance (Janzen and van Turennout, 2004), immobility (Mullally and Maguire, 2011), or larger physical size (Troiani et al., 2014). Consistent with these previous reports, we find that responses measured in three scene-selective areas carry substantial information about visual object categories, and that these responses are increased in the presence of topographical elements, such as buildings or roads, which carry cues about the spatial environment. However, we also find that all ROIs yield greater responses for man-made instruments and vehicles than for large, stationary objects, such as structures or geological formations. Thus, our results imply that during natural vision, category representation in scene-selective ROIs is not strictly constrained by physical size or level of mobility.

That said, it remains an open question whether scene-selective ROIs represent categories exclusively, or rather other visual features that might be systematically related to categories. In a recent study, we compared the predictive performance of Fourier power, subjective distance, and object category models in the PPA, RSC, and OPA using BOLD responses to natural images (Lescroart et al., 2015). In that study we found that a significant portion of the response variance explained by these three models is shared. This unanticipated result is likely caused by the intrinsic correlations between visual features in natural scenes. We were therefore concerned that our current results might have been biased by the correlation between low-level features and categories. To ensure that this was not the case, we ran separate control analyses demonstrating that neither category tuning nor its heterogeneous distribution within scene-selective areas can be explained by tuning for low-level features, including spatial texture, 
OPA1
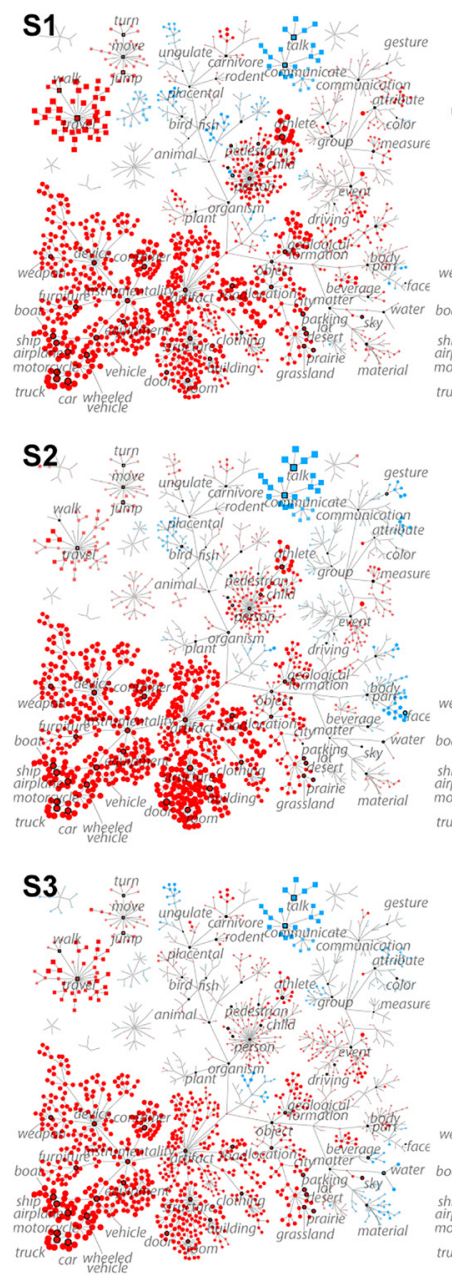

OPA2
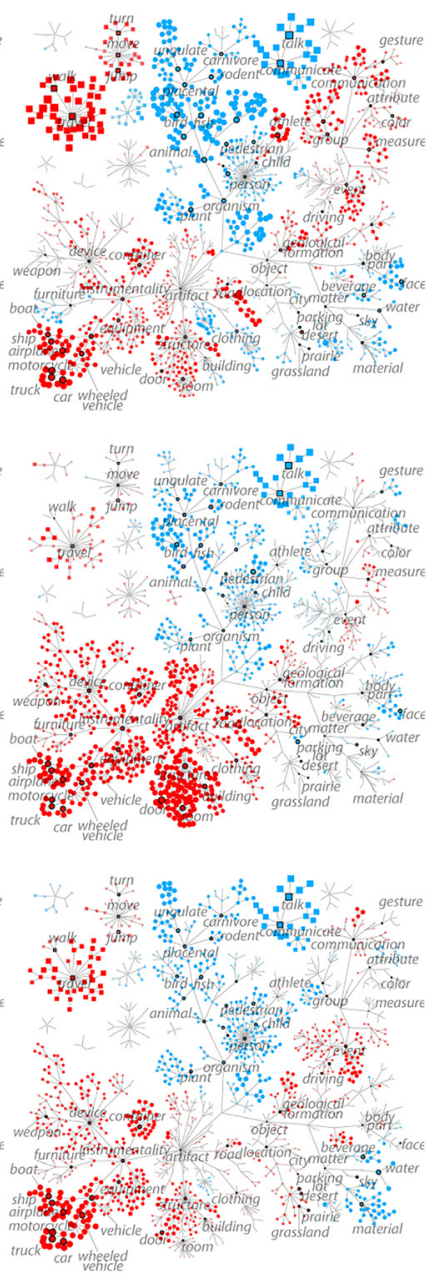

OPA1
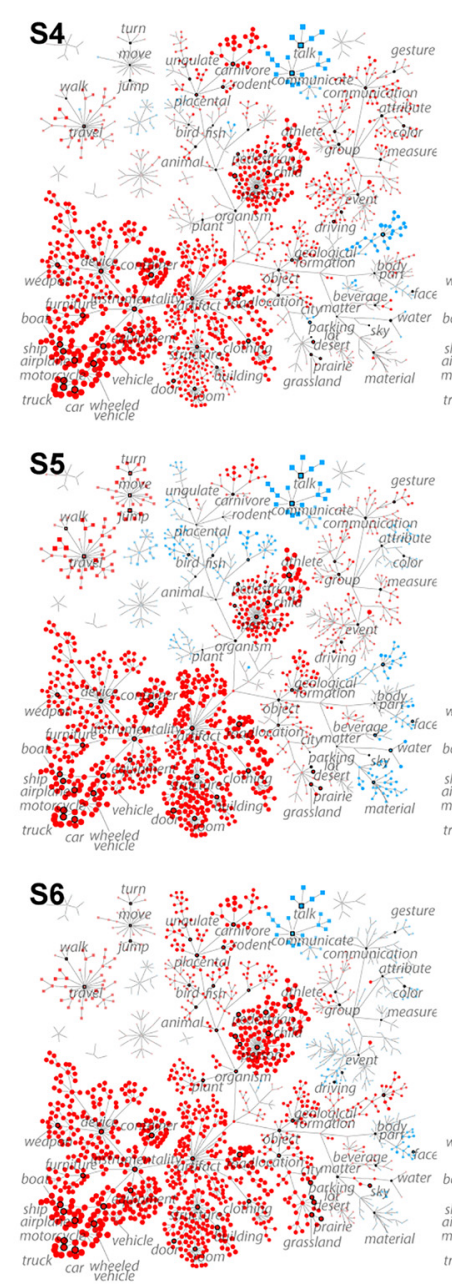

OPA2
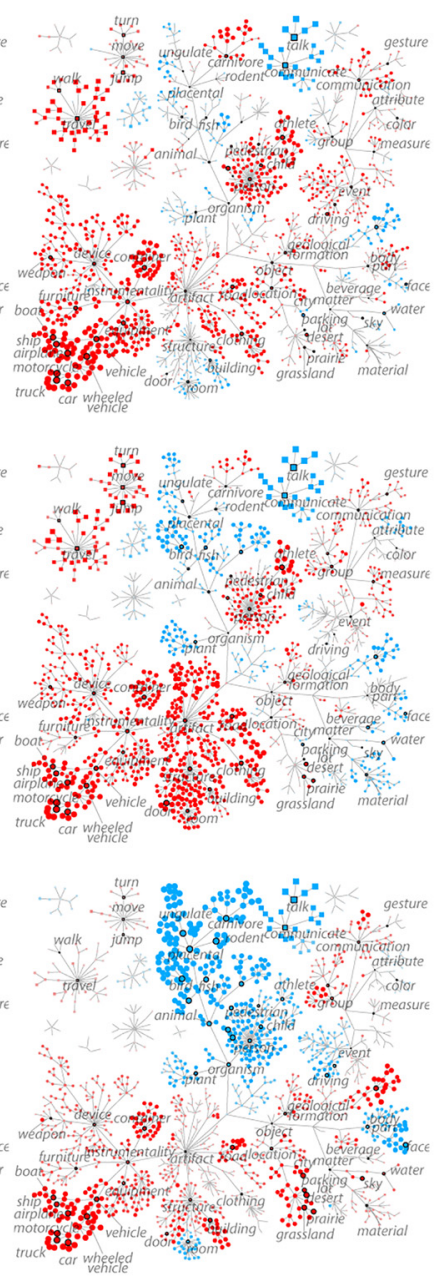

Figure 14. Individual-subject cluster centers in the OPA. Cluster analysis was performed separately in individual subjects (data are aggregated across hemispheres). The left and right panels for each subject show the centers of the two clusters in OPA1 and OPA2. The center of each cluster was taken as the mean tuning profile across voxels in that cluster. Tuning diagrams formatted as in Figure 5. The cluster centers are highly consistent across individual subjects.

layout, and spatiotemporal structure. However, previous studies have argued that these areas also represent intermediate features, such as subjective spatial distance (Lescroart et al., 2015), spatial expanse (Op de Beeck et al., 2008; Kravitz et al., 2011b), spacedefining properties (Mullally and Maguire, 2011), or contextual associations (Bar et al., 2008). Thus it is possible that the two subdivisions in the PPA, RSC, and OPA are differentially tuned for such features, and these tuning differences underlie the distinctive responses of the subdivisions to object/action categories. Further research on this challenging question is warranted.

The natural movie stimulus used in this study depicts thousands of object and action categories as they appear in the real world. Thus, another stimulus-related concern was that our results might have been biased by the inherent correlations among these categories. To preclude this possibility, we ran a control analysis indicating that tuning for multiple objects and actions measured in the PPA, RSC, and OPA is not biased by correlations between distinct categories in the finite movie stimulus. However, our control analysis does not discern whether these areas represent individual objects or statistical ensembles of objects within visual scenes (Stansbury et al., 2013). A recent study from our laboratory suggests that the highly selective and nonlinear tuning of scene-selective areas causes them to respond to higher- order correlations in natural images (Lescroart et al., 2015). As it is difficult (perhaps impossible) to create stimuli in which these correlations are completely removed, it is inherently challenging to adjudicate between alternatives, such as an object category model and a model that describes object co-occurrence statistics. However, future studies may shed light on this problem by compiling a more controlled set of natural stimuli that minimizes category co-occurrence while retaining a reasonable range of variance in individual categories.

\section{Functional similarity of category representation across the PPA, RSC, and OPA}

Functional differences among the PPA, RSC, and OPA have been investigated in several previous studies. For example, Epstein et al. (2007) reported that differential responses to images of familiar versus unfamiliar locations are stronger in the RSC relative to the PPA and OPA. In a separate study Epstein and Higgins (2007) measured differential responses to static scenes viewed during either location-identification or category-identification tasks and reported larger task-related differences in the RSC than in the PPA. These results have led to the view that the PPA and OPA likely represent physical stimulus attributes, whereas the RSC primarily represents spatial and contextual associations of the 
local stimulus to the extended environment (Epstein, 2008). In contrast, we observe a surprisingly similar pattern of functional tuning across the three scene-selective ROIs (Figs. 5, 6). Several methodological differences between our study and some previous studies may account for this apparent difference. First, while many previous studies used an explicit identification task or they manipulated scene familiarity, here we used passive fixation. Second, while previous studies primarily measured selectivity for spatial stimulus attributes, here we focused on category representations that are mostly invariant to spatial factors (DiCarlo et al., 2012). Therefore, our results could be taken to imply that functional differences among scene-selective ROIs are task-dependent, and that they are relatively weaker for high-level category representations.

\section{Spatial heterogeneity of category tuning in the PPA, RSC, and OPA}

Several recent studies have investigated the spatial distribution of selectivity for high-level and low-level visual features across the PPA (Arcaro et al., 2009; Rajimehr et al., 2011; Baldassano et al., 2013). In an earlier study, Arcaro et al. (2009) identified a cortical region that overlaps with the posterior PPA and yields a stronger scenes-objects contrast than does a neighboring region that overlaps with the anterior PPA. Baldassano et al. (2013) later suggested that the posterior PPA yields stronger responses to scenes and abstract objects than does the anterior PPA. It has also been reported by Rajimehr et al. (2011) that a lateral-posterior patch within the PPA responds preferentially to high spatial frequencies, while remaining parts of the PPA do not show significant frequency bias. Together these previous findings imply that representations of both categorical and lower-level visual features might be weaker in anterior parts of the PPA compared with the posterior PPA.

The posterior subregions within the PPA previously suggested to show stronger category and spatial-frequency tuning partly overlap with the first subdomain (PPA1) identified here, which is tuned for man-made artifacts in static scenes. However, the second subdomain (PPA2) that we identify, located anteriomedially, is also significantly selective for many object and action categories related to navigation. Furthermore, we find no significant differences in spatial-frequency tuning between the two subdomains. Thus, it appears that measured differences in category tuning cannot be attributed to a mere spatial frequency bias. In contrast to previous studies that used static stimuli containing isolated objects (Arcaro et al., 2009; Rajimehr et al., 2011; Baldassano et al., 2013), our study used dynamic natural movies. Thus, the relatively weaker category selectivity in the anterior PPA that was reported previously might merely reflect an experimental bias due to the use of static stimuli, which contain relatively fewer objects and actions clearly related to navigation.

In summary we identify two subregions in each sceneselective area, the PPA, RSC and OPA: one primarily selective for categories of inanimate, man-made objects encountered frequently in daily life, and another selective for vehicles and locomotion-related action categories that appear in dynamic scenes. Spatial segregation of selectivity for objects and actions that appear in static versus dynamic visual scenes has overarching implications for the cortical organization of category representation. Scene-selective areas in humans and homologous areas in monkeys have been shown to share functional properties with visual areas along both the dorsal and ventral pathways (Kravitz et al., 2011a). It is thus likely that heterogeneous category tuning in scene-selective areas might be a reflection of the functional division between dorsal and ventral visual pathways (Ungerleider and Haxby, 1994; Shmuelof and Zohary, 2005). This view suggests that there might be two separate and parallel processing streams passing through scene-selective ROIs, one biased toward static stimuli (i.e., low temporal frequency) and one biased toward moving stimuli (i.e., high temporal frequency). While the former stream might be critical for navigation within the extended spatial environment, the latter may play a role in avoiding mobile obstacles.

\section{References}

Arcaro MJ, McMains SA, Singer BD, Kastner S (2009) Retinotopic organization of human ventral visual cortex. J Neurosci 29:10638-10652. CrossRef Medline

Baldassano C, Beck DM, Fei-Fei L (2013) Differential connectivity within the parahippocampal place area. Neuroimage 75:228-237. CrossRef Medline

Bar M (2004) Visual objects in context. Nat Rev Neurosci 5:617-629. CrossRef Medline

Bar M, Aminoff E, Schacter DL (2008) Scenes unseen: the parahippocampal cortex intrinsically subserves contextual associations, not scenes or places per se. J Neurosci 28:8539-8544. CrossRef Medline

Ben-Hur A, Elisseeff A, Guyon I (2002) A stability based method for discovering structure in clustered data. Pac Symp Biocomp 6-17. Medline

Benjamini Y, Yekutieli D (2001) The control of the false discovery rate in multiple testing under dependency. Ann Stat 29:1165-1188. CrossRef

Cant JS, Goodale MA (2011) Scratching beneath the surface: new insights into the functional properties of the lateral occipital area and parahippocampal place area. J Neurosci 31:8248-8258. CrossRef Medline

Connolly JD, Goodale MA, DeSouza JF, Menon RS, Vilis T (2000) A comparison of frontoparietal fMRI activation during anti-saccades and antipointing. J Neurophysiol 84:1645-1655. Medline

Çukur T, Nishimoto S, Huth AG, Gallant JL (2013a) Attention during natural vision warps semantic representation across the human brain. Nat Neurosci 16:763-770. CrossRef Medline

Çukur T, Huth AG, Nishimoto S, Gallant JL (2013b) Functional subdomains within human FFA. J Neurosci 33:16748-16766. CrossRef Medline

DiCarlo JJ, Zoccolan D, Rust NC (2012) How does the brain solve visual object recognition? Neuron 73:415-434. CrossRef Medline

Dilks DD, Julian JB, Paunov AM, Kanwisher N (2013) The occipital place area is causally and selectively involved in scene perception. J Neurosci 33:1331-1336a. CrossRef Medline

Downing PE, Chan AW, Peelen MV, Dodds CM, Kanwisher N (2006) Domain specificity in visual cortex. Cereb Cortex 16:1453-1461. Medline

Engel SA, Glover GH, Wandell BA (1997) Retinotopic organization in human visual cortex and the spatial precision of functional MRI. Cereb Cortex 7:181-192. CrossRef Medline

Epstein RA, Higgins JS (2007) Differential parahippocampal and retrosplenial involvement in three types of visual scene recognition. Cereb Cortex 17:1680-1693. CrossRef Medline

Epstein RA, Higgins JS, Jablonski K, Feiler AM (2007) Visual scene processing in familiar and unfamiliar environments. J Neurophysiol 97:36703683. CrossRef Medline

Epstein RA (2008) Parahippocampal and retrosplenial contributions to human spatial navigation. Trends Cogn Sci 12:388-396. CrossRef Medline

Epstein R, Kanwisher N (1998) A cortical representation of the local visual environment. Nature 392:598-601. CrossRef Medline

Field DJ (1987) Relations between the statistics of natural images and the response properties of cortical cells. J Opt Soc Am A 4:2379-2394. CrossRef Medline

Friston KJ, Rotshtein P, Geng JJ, Sterzer P, Henson RN (2006) A critique of functional localisers. Neuroimage 30:1077-1087. CrossRef Medline

Geisler WS (2008) Visual perception and the statistical properties of natural scenes. Annu Rev Psychol 59:167-192. CrossRef Medline

Grill-Spector K, Malach R (2004) The human visual cortex. Annu Rev Neurosci 27:649-677. CrossRef Medline

Grill-Spector K, Sayres R, Ress D (2006) High-resolution imaging reveals highly selective nonface clusters in the fusiform face area. Nat Neurosci 9:1177-1185. CrossRef Medline

Handl J, Knowles J, Kell DB (2005) Computational cluster validation in 
post-genomic data analysis. Bioinformatics 21:3201-3212. CrossRef Medline

Hansen KA, Kay KN, Gallant JL (2007) Topographic organization in and near human visual area V4. J Neurosci 27:11896-11911. CrossRef Medline

Haxby JV, Gobbini MI, Furey ML, Ishai A, Schouten JL, Pietrini P (2001) Distributed and overlapping representations of faces and objects in ventral temporal cortex. Science 293:2425-2430. CrossRef Medline

Haxby JV, Guntupalli JS, Connolly AC, Halchenko YO, Conroy BR, Gobbini MI, Hanke M, Ramadge PJ (2011) A common, high-dimensional model of the representational space in human ventral temporal cortex. Neuron 72:404-416. CrossRef Medline

Huth AG, Nishimoto S, Vu AT, Gallant JL (2012) A continuous semantic space describes the representation of thousands of object and action categories across the human brain. Neuron 76:1210-1224. CrossRef Medline

Jaccard P (1908) Nouvelles recherches sur la distribution florale. Bull Soc Vaud Sci Nat 44:223-270.

Janzen G, van Turennout M (2004) Selective neural representation of objects relevant for navigation. Nat Neurosci 7:673-677. CrossRef Medline

Jenkinson M, Bannister P, Brady M, Smith S (2002) Improved optimization for the robust and accurate linear registration and motion correction of brain images. Neuroimage 17:825-841. CrossRef Medline

Kay KN, Naselaris T, Prenger RJ, Gallant JL (2008) Identifying natural images from human brain activity. Nature 452:352-355. CrossRef Medline

Kravitz DJ, Saleem KS, Baker CI, Mishkin M (2011a) A new neural framework for visuospatial processing. Nat Rev Neurosci 12:217-230. CrossRef Medline

Kravitz DJ, Peng CS, Baker CI (2011b) Real-world scene representations in high-level visual cortex: it's the spaces more than the places. J Neurosci 31:7322-7333. CrossRef Medline

Lescroart MD, Stansbury DE, Gallant JL (2015) Fourier power, subjective distance, and object categories all provide plausible models of BOLD responses in scene-selective visual areas. Front Comput Neurosci 9:135. CrossRef Medline

MacEvoy SP, Epstein RA (2007) Position selectivity in scene- and objectresponsive occipitotemporal regions. J Neurophysiol 98:2089-2098. CrossRef Medline

Macevoy SP, Epstein RA (2009) Decoding the representation of multiple simultaneous objects in human occipitotemporal cortex. Curr Biol 19: 943-947. CrossRef Medline

MacEvoy SP, Epstein RA (2011) Constructing scenes from objects in human occipitotemporal cortex. Nat Neurosci 14:1323-1329. CrossRef Medline

Miller G (1995) WordNet: a lexical database for English. Commun ACM 38:39-41. CrossRef

Mullally SL, Maguire EA (2011) A new role for the parahippocampal cortex in representing space. J Neurosci 31:7441-7449. CrossRef Medline

Nasr S, Liu N, Devaney KJ, Yue X, Rajimehr R, Ungerleider LG, Tootell RB (2011) Scene-selective cortical regions in human and nonhuman primates. J Neurosci 31:13771-13785. CrossRef Medline

Ng AY, Jordan MI, Weiss Y (2001) On spectral clustering: analysis and an algorithm. In: Advances in Neural Information Processing Systems (Dietterich T, Becker S, Ghahramani Z, eds), pp 849-856. MIT, Cambridge.

Nishimoto S, Vu AT, Naselaris T, Benjamini Y, Yu B, Gallant JL (2011) Reconstructing visual experiences from brain activity evoked by natural movies. Curr Biol 21:1641-1646. CrossRef Medline
Oliva A, Torralba A (2001) Modeling the shape of the scene: a holistic representation of the spatial envelope. Int J Comput Vis 42:145-175.

Op de Beeck HP, Haushofer J, Kanwisher NG (2008) Interpreting fMRI data: maps, modules and dimensions. Nat Rev Neurosci 9:123-135. CrossRef Medline

Park S, Intraub H, Yi DJ, Widders D, Chun MM (2007) Beyond the edges of a view: boundary extension in human scene-selective visual cortex. Neuron 54:335-342. CrossRef Medline

Park S, Brady TF, Greene MR, Oliva A (2011) Disentangling scene content from spatial boundary: complementary roles for the parahippocampal place area and lateral occipital complex in representing real-world scenes. J Neurosci 31:1333-1340. CrossRef Medline

Rajimehr R, Devaney KJ, Bilenko NY, Young JC, Tootell RB (2011) The "parahippocampal place area" responds preferentially to high spatial frequencies in humans and monkeys. PLoS Biol 9:e1000608. CrossRef Medline

Reddy L, Kanwisher N (2007) Category selectivity in the ventral visual pathway confers robustness to clutter and diverted attention. Curr Biol 17: 2067-2072. CrossRef Medline

Rossion B, Dricot L, Devolder A, Bodart JM, Crommelinck M, De Gelder B, Zoontjes R (2000) Hemispheric asymmetries for whole-based and partbased face processing in the human fusiform gyrus. J Cogn Neurosci 12:793-802. CrossRef Medline

Shmuelof L, Zohary E (2005) Dissociation between ventral and dorsal fMRI activation during object and action recognition. Neuron 47:457-470. CrossRef Medline

Spiridon M, Fischl B, Kanwisher N (2006) Location and spatial profile of category-specific regions in human extrastriate cortex. Hum Brain Mapp 27:77-89. CrossRef Medline

Stansbury DE, Naselaris T, Gallant JL (2013) Natural scene statistics account for the representation of scene categories in human visual cortex. Neuron 79:1025-1034. CrossRef Medline

Stevens WD, Kahn I, Wig GS, Schacter DL (2012) Hemispheric asymmetry of visual scene processing in the human brain: evidence from repetition priming and intrinsic activity. Cereb Cortex 22:1935-1949. CrossRef Medline

Tootell RB, Reppas JB, Kwong KK, Malach R, Born RT, Brady TJ, Rosen BR, Belliveau JW (1995) Functional analysis of human MT and related visual cortical areas using magnetic resonance imaging. J Neurosci 15:32153230. Medline

Troiani V, Stigliani A, Smith ME, Epstein RA (2014) Multiple object properties drive scene-selective regions. Cereb Cortex 24:883-897. CrossRef Medline

Ungerleider LG, Haxby JV (1994) 'What' and 'where' in the human brain. Curr Opin Neurobiol 4:157-165. CrossRef Medline

Van Essen DC, Drury HA, Dickson J, Harwell J, Hanlon D, Anderson CH (2001) An integrated software suite for surface-based analyses of cerebral cortex. J Am Med Inform Assoc 8:443-459. CrossRef Medline

Vinje WE, Gallant JL (2000) Sparse coding and decorrelation in primary visual cortex during natural vision. Science 287:1273-1276. CrossRef Medline

Walther DB, Caddigan E, Fei-Fei L, Beck DM (2009) Natural scene categories revealed in distributed patterns of activity in the human brain. J Neurosci 29:10573-10581. CrossRef Medline

Walther DB, Chai B, Caddigan E, Beck DM, Fei-Fei L (2011) Simple line drawings suffice for functional MRI decoding of natural scene categories. Proc Natl Acad Sci U S A 108:9661-9666. CrossRef Medline 